\title{
Semantic Tableau Versions of Some Normal Modal Systems with Propositional Quantifiers
}

\author{
Daniel Rönnedal ${ }^{*}$
}

Received: 12 July 2018 / Accepted: 2 January 2019

Abstract: In Symbolic Logic (1932), C. I. Lewis developed five modal systems $S 1-S 5$. $S 4$ and $S 5$ are so-called normal modal systems. Since Lewis and Langford's pioneering work many other systems of this kind have been investigated, among them the 32 systems that can be generated by the five axioms $T, D, B, 4$ and 5. Lewis also discusses how his systems can be augmented by propositional quantifiers and how these augmented logics allow us to express some interesting ideas that cannot be expressed in the corresponding quantifier-free logics. In this paper, I will develop 64 normal modal semantic tableau systems that can be extended by propositional quantifiers yielding 64 extended systems. All in all, we will investigate 128 different systems. I will show how these systems can be used to prove some interesting theorems and I will discuss Lewis's so-called existence postulate and some of its consequences. Finally, I will prove that all normal modal systems are sound and complete and that all systems (including the extended systems) are sound with respect to their semantics. It is left as an open question whether or not the extended systems are complete.

Keywords: C. I. Lewis, Modal logic, Propositional quantifiers, Semantic tableaux.

*Stockholm University

Department of Philosophy, University of Stockholm, Universitetsvägen 10 D, 10691 Stockholm, Sweden

\ daniel.ronnedal@philosophy.su.se

(C) The Author. Journal compilation (C) The Editorial Board, Organon F. 


\section{Introduction}

Modal logic deals with modal concepts, such as necessity, possibility and contingency, and with the logical relationships between propositions that include such concepts. Modal logicians study various modal principles, arguments and systems (Blackburn et. al. 2001; 2007, Chellas 1980, Fitting and Mendelsohn 1998, Garson 2006, Hughes and Cresswell 1968; 1996, Kracht 1999, Priest 2008). Lewis and Langford's Symbolic Logic (1932) marks the beginning of modern, symbolic, modal logic.

The purpose of this paper is to develop 64 so-called normal modal semantic tableau systems (half of them correspond to the 32 axiomatic systems that can be generated by the well-known axioms $T, D, B, 4$ and 5) and to show how these systems can be augmented by propositional quantifiers. Therefore, we will consider 128 different systems in this paper. The tableau rules $T-T$, $T-D, T-B, T-4$ and $T-5$ and the 32 systems that can be generated from these rules are well-known. The normal modal tableau system that includes $T-T$ and $T-4$ is deductively equivalent with, that is, includes the same theorems as, Lewis's system $S 4$, and the normal modal tableau system that includes $T-T, T-B$ and $T-4$ is deductively equivalent with Lewis's system $S 5 .^{1}$ All systems in this paper are stronger than Lewis's systems $S 1-$ $S 3$. The tableau rule $T-F$, which is especially interesting for our purposes, is much less well-known. Hence, all systems that contain this rule deserve extra attention. The propositional part of all systems in this paper is fairly standard, but as far as I know there are no modal tableau systems in the literature that include propositional quantifiers of the kind that is used in our formal language. ${ }^{2}$ Hence, all 64 extended systems are new. Furthermore, I will show how these systems can be used to prove some interesting theorems that contain propositional quantifiers and I will discuss Lewis's so-called existence postulate and some of its consequences. According to this postulate, there is some pair of propositions $X$ and $Y$, so related that $X$ implies nothing about the truth or falsity of $Y$ (Lewis and Langford 1932, 179). This postulate can be symbolised in the following way: $\exists X \exists Y(\neg \square(X \rightarrow Y) \wedge \neg \square(X \rightarrow \neg Y))$. Finally, I will prove that all normal modal systems are sound and complete

\footnotetext{
${ }^{1}$ In a strict sense this proposition is not true, since I will not use the exact same language as Lewis. However, if we were to use the same language, they would be deductively equivalent. I will ignore such 'trivial' differences in this paper.

${ }^{2}$ However, (Bull 1969) and (Kripke 1959) use tableaux that are vaguely similar to the tableaux I use in this paper.
}

Organon F 26 (3) 2019: 505-536 
and that all systems (including the extended systems) are sound with respect to their semantics. It is left as an open question whether or not the extended systems are complete.

Since all extended systems in this paper are new, there are good logical reasons to be interested in our results. There are also several philosophically interesting reasons. In systems with propositional quantifiers we can express many ideas that cannot be expressed in any quantifier-free normal modal systems. We can, for example, symbolise Lewis's existence postulate, from which it follows that there is something that is contingent, that material implication does not coincide with necessary implication, and that there are at least four distinct propositions, among other things. In ordinary normal modal systems, we cannot prove any of these propositions; we cannot even find plausible formalisations of them. Furthermore, the tableau systems are often more user-friendly than their axiomatic counterparts, it is often easier to prove something in a tableau system than in an axiomatic system and it is often easier to derive a sentence from a set of premises. Consequently, there are both good philosophical and technical reasons to be interested in the systems in this paper. (For more information on propositional quantifiers, see, for example (Lewis and Langford 1932, 178-198), (Kripke 1959), (Bull 1969), (Fine 1970), (Kaplan 1970), (Gabbay 1971) and (Gallin 1975).)

\section{Syntax}

First, we introduce a quantifier-free language. Then we extend this language with propositional quantifiers.

Alphabet. A set of propositional variables $P, Q, R, S, T, X, Y, Z, W$, $P_{1}, Q_{1}, R_{1}, S_{1}, T_{1}, X_{1}, Y_{1}, Z_{1}, W_{1}, P_{2}, Q_{2}, R_{2}, S_{2}, T_{2}, X_{2}, Y_{2}, Z_{2}, W_{2}$, $\ldots ; \perp$ (Falsum) and $T$ (Verum); primitive truth-functional connectives: $\neg$ (negation), $\wedge$ (conjunction),$\vee$ (disjunction), $\rightarrow$ (material implication), and $\leftrightarrow$ (material equivalence); modal operators: $\square$ (necessity) and $\diamond$ (possibility), and (iv) the brackets ), (.

The language $\mathcal{L}$. The language $\mathcal{L}$ is the set of formulas generated by the usual clauses for atomic sentences, Verum and Falsum and propositionally compound sentences, and the following clause: if $A$ is a formula, then $\square A$ 
(it is necessary that $A$ ) and $\diamond A$ (it is possible that $A$ ) are formulas; nothing else is a formula. $A, B, C, \ldots$ stand for arbitrary formulas. Formulas are also called 'sentences'.

Definitions. $A$ (it is impossible that $A)={ }_{d f} \neg \diamond A$ (or $\square \neg A$ ); $\square A$ (it is nonnecessary that $A)={ }_{d f} \neg \square A ; \nabla A$ (it is contingent that $\left.A\right)=_{d f}(\diamond A \wedge \diamond \neg A) ; \boldsymbol{\nabla} A$ (it is non-contingent that $A)={ }_{d f} \neg \nabla A($ or $(\square A \vee \square \neg A)$ ); $(A \circ B)(A$ is consistent with $B)={ }_{d f} \triangleright(A \wedge B) ;(A \bullet B)(A$ is inconsistent with $B)={ }_{d f} \neg(A \circ B)$ $(\neg \diamond(A \wedge B), \diamond(A \wedge B)$, or $\square \neg(A \wedge B)),(A \Rightarrow B)(A$ strictly implies $B)$ $={ }_{d f} \square(A \rightarrow B),(A \Leftrightarrow B)(A$ is strictly equivalent with $B)={ }_{d f}((\mathrm{~A} \Rightarrow B) \wedge(B \Rightarrow$ $A))(((\square(A \rightarrow B) \wedge \square(B \rightarrow A))$, or $\square(A \leftrightarrow B))$.

The language $\mathcal{L}_{\text {Ext }}$. We extend $\mathcal{L}$ by adding two propositional quantifiers $\forall$ (everything/for all) and $\exists$ (something/for some) in the usual way. So, if $A$ is any formula and $X$ is any propositional variable, then $\forall X A$ and $\exists X A$ are formulas. We will call this extended language $\mathcal{L}_{E x t}$. Parentheses are usually dropped when it does not lead to any ambiguity.

The concept of a free variable $X$ in a formula $A$ is defined in the usual way. A variable $X$ is free in $A$ if and only if (iff) it has a free occurrence in $A$. Intuitively, an occurrence of a variable is free in a formula just in case it is not bound by any quantifier. If $A$ does not contain $\forall$ or $\exists$, then every occurrence of $X$ in $A$ is free. An occurrence of $X$ is free in $\neg B(\square B, \diamond B)$ iff the corresponding occurrence of $X$ is free in $B$, and an occurrence of $X$ in $A \wedge B$ is free iff the corresponding occurrence of $X$ in $A$ or $B$ is free, etc. Finally, an occurrence of $X$ is free in $\forall Y B(\exists Y B)$ iff the corresponding occurrence of $X$ is free in $B$ and $X$ is distinct from $Y$. Any variable occurrences in a formula that are not free are said to be bound. Every free occurrence of $X$ in $B$ is bound by $\forall(\exists)$ in $\forall X B(\exists X B)$.

Let $(A)\left[B_{1} / X_{1}, \ldots, B_{n} / X_{n}\right]$ be the formula that results by simultaneously replacing all free occurrences of the variable $X_{1}$ in $A$ by $B_{1}, \ldots$, and all free occurrences of the variable $X_{n}$ in $A$ by $B_{n}$. If there are no free occurrences of $X_{1}, \ldots, X_{n}$ in $A$, then $(A)\left[B_{1} / X_{1}, \ldots, B_{n} / X_{n}\right]=A$. We say that $B$ is substitutable for $X$ in $A$ just in case no variable in $B$ gets bound by a quantifier when $B$ is substituted for $X$ in $A$. In other words, $B$ is substitutable for $X$ in $A$ just in case, for every variable $Y$, if an occurrence of $Y$ is free in $B$, then the corresponding occurrence of $Y$ is free when $X$ is replaced by $B$ in $A$. 
Lewis uses the identity sign for necessary equivalence since he thinks that propositions that are necessarily equivalent are identical. We shall also say that two 'propositions' are 'identical' iff they are necessarily equivalent and that they are 'distinct' iff they are not necessarily equivalent. So, $\exists X \exists Y \neg(X \Leftrightarrow$ $Y$ ) says that there are two distinct propositions.

\section{Semantics}

Model. A model $\mathcal{M}$ is a structure $\langle\mathfrak{W}, \mathfrak{R}, \mathfrak{v}\rangle$, where $\mathfrak{W}$ is a (non-empty) set of possible worlds, $\mathfrak{R}$ is a binary accessibility relation between possible worlds, and $\mathfrak{v}$ is a valuation function. Intuitively, ' $\mathfrak{R} \omega \omega^{\prime}$ ' means that the possible world $\omega^{\prime}$ is accessible from the possible world $\omega$, or that $\omega$ 'can see' $\omega^{\prime}$. Let $X$ be a propositional variable. Then $\mathfrak{v}(X)$ is a subset of $\mathfrak{W}$. Intuitively, $\mathfrak{v}(X)$ is the proposition that $X$ expresses or the set of possible worlds in which $X$ is true.

Truth conditions. The truth conditions for sentences in $\mathcal{L}$ are defined in the usual way. ' $\mathcal{M}, \omega \Vdash A$ ' says that $A$ is true in the possible world $\omega$ in the model $\mathcal{M}$. If $X$ is a propositional variable, then $\mathcal{M}, \omega \Vdash X$ iff $\omega$ is an element in $\mathfrak{v}(X)$. Verum is true in every possible world in every model and Falsum is false in every possible world in every model. $\mathcal{M}, \omega \Vdash \neg A$ iff it is not the case that $\mathcal{M}, \omega \Vdash A$. $\mathcal{M}, \omega \Vdash A \wedge B$ iff $\mathcal{M}, \omega \Vdash A$ and $\mathcal{M}, \omega \Vdash B$, etc. $\mathcal{M}, \omega \Vdash \square A$ iff for every $\omega^{\prime}$ in $\mathfrak{W}$ such that $\mathfrak{R} \omega \omega^{\prime}: \mathcal{M}, \omega^{\prime} \Vdash A$. $\mathcal{M}, \omega \Vdash \diamond A$ iff for some $\omega^{\prime}$ in $\mathfrak{W}: \mathfrak{R} \omega \omega^{\prime}$ and $\mathcal{M}, \omega^{\prime} \Vdash A$.

Intuitively, $\forall X A$ is true in a possible world $\omega$ iff $A[B / X]$ is true in $\omega$ for every sentence $B$ in $\mathcal{L}$, and $\exists X A$ is true in $\omega$ iff $A[B / X]$ is true in $\omega$ for some sentence $B$ in $\mathcal{L}$. So, the quantifiers are 'substitutional' rather than 'objectual'. For example, they do not vary directly over (sets of) possible worlds (the range is not a (the) set of (all) subsets of (the set of all) possible worlds). To avoid circularity, we only use formulas from $\mathcal{L}$ in our substitutions. To see the potential problem, let $A=\forall X X$ and assume that our substitutions can include any formula whatsoever. Then $A[A / X]=A$, for $\forall X X[\forall X X / X]=\forall X X$. More precisely, the truth conditions for the new sentences in $\mathcal{L}_{E x t}$ are defined in the following way: $\mathcal{M}, \omega \Vdash \forall X A$ iff for every sentence $B$ (that is substitutable for $X$ in $A$ ) in $\mathcal{L}, \mathcal{M}, \omega \Vdash A[B / X] . \mathcal{M}, \omega \Vdash \exists X A$ iff there is some sentence $B$ 
(that is substitutable for $X$ in $A$ ) in $\mathcal{L}$ such that $\mathcal{M}, \omega \Vdash A[B / X]{ }^{3}$

Validity. A sentence $A$ is valid in a model, $\mathcal{M} \Vdash A$, iff $A$ is true in every possible world $\omega$ in $\mathcal{M}$. Let $\mathfrak{M}$ be a class of models. Then $A$ is valid in $\mathfrak{M}$, $\mathfrak{M} \Vdash A$, iff $A$ is valid in every model $\mathcal{M}$ in $\mathfrak{M}$, that is, iff $A$ is true in every possible world $\omega$ in every model $\mathcal{M}$ in $\mathfrak{M}$.

Logical consequence. Let $A$ be a sentence, let $\Gamma$ be a finite set of sentences and let $\mathfrak{M}$ be a class of models. Then, $A$ is a logical consequence of $\Gamma$ in $\mathfrak{M}, \mathfrak{M}, \Gamma \Vdash A$, iff for every model $\mathcal{M}$ in $\mathfrak{M}$ and world $\omega$ in $\mathcal{M}$, if all elements of $\Gamma$ are true in $\omega$ in $\mathcal{M}$, then $A$ is true in $\omega$ in $\mathcal{M}$. If $\mathfrak{M}, \Gamma \Vdash A$, we also say that $\Gamma$ entails $A$ in $\mathfrak{M}$ and that the argument from $\Gamma$ to $A$ is valid in $\mathfrak{M}$. An argument is invalid in $\mathfrak{M}$ iff it is not valid in $\mathfrak{M}$.

\subsection{Conditions on models}

In this section, I will consider some conditions that might be imposed on the accessibility relation in a model. In Figure 1, I have listed some of the most well-known conditions in the literature on modal logic along with some formulas. If a model $\mathcal{M}$ satisfies $C-T$, then $T$ is valid in $\mathcal{M}$; if a model $\mathcal{M}$ satisfies $C-D$, then $D$ is valid in $\mathcal{M}$, etc. $C-T, C-D, C-B, C-4$ and $C-5$ are mentioned in most introductions to modal logic and they require no further discussion. Condition $C-F$ is less well-known. It is especially interesting for our purposes in this paper. We will, for example, see that this condition is incompatible with Lewis's existence postulate.

The conditions mentioned in Figure 1 can be used to obtain a categorisation of the set of all models into various kinds. We shall say that $\mathfrak{M}\left(C_{1}, \ldots, C_{n}\right)$ is the class of all models that satisfy the conditions $C_{1}, \ldots, C_{n}$. For example, $\mathfrak{M}(C-T, C-B, C-4)$ is the class of all models that satisfy the conditions $C-T, C-B$ and $C-4$.

\footnotetext{
${ }^{3}$ Some of the systems in this paper are vaguely similar to some systems mentioned by (Bull 1969) and (Kripke 1959). There are also some similarities between propositional quantification and quantification over individuals. In Section 4.2, we will see that some Barcan-like formulas can be proved in all systems in this paper. This means that the propositional quantifiers act more like so-called possibilist quantifiers than like socalled actualist quantifiers. For more on how to combine modal logic with predicate logic where the quantifiers vary over objects, see, for example, (Barcan 1946), (Carnap 1946), (Corsi 2002), (Fitting and Mendelsohn 1998), (Garson 1984; 2006), (Hintikka 1961), (Hughes and Cresswell 1968; 1996), (Parks 1976), (Priest 2008), (Stalnaker and Thomason 1968), (Thomason 1970) and (Thomason and Stalnaker 1968).
} 


\begin{tabular}{l|l|l|l}
\hline & Conditions on $\mathfrak{R}$ & & Corresponding formulas \\
\hline$C-K$ & - & $K$ & $\square(P \rightarrow Q) \rightarrow(\square \rightarrow \square Q)$ \\
$C-T$ & $\forall x \mathfrak{R} x x$ & $T$ & $\square P \rightarrow P$ \\
$C-D$ & $\forall x \exists y \mathfrak{R} x y$ & $D$ & $\square P \rightarrow \diamond P$ \\
$C-B$ & $\forall x \forall y(\mathfrak{R} x y \rightarrow \mathfrak{R} y x)$ & $B$ & $P \rightarrow \square \diamond P$ \\
$C-F$ & $\forall x \forall y \forall z((\mathfrak{R} x y \wedge \mathfrak{R} x z) \rightarrow y=z)$ & $F$ & $\diamond P \rightarrow \square P$ \\
$C-4$ & $\forall x \forall y \forall z((\mathfrak{R} x y \wedge \mathfrak{R} y z) \rightarrow \mathfrak{R} x)$ & 4 & $\square P \rightarrow \square \square P$ \\
$C-5$ & $\forall x \forall y \forall z((\mathfrak{R} x y \wedge \mathfrak{R} x z) \rightarrow \mathfrak{R} y z)$ & 5 & $\diamond P \rightarrow \square \diamond P$ \\
\hline
\end{tabular}

Figure 1: Conditions on models

By using this classification of model classes we can define a large set of systems. The set of all sentences in a language that are valid in a class of models $\mathfrak{M}$ is the (logical) system of $\mathfrak{M}, S(\mathfrak{M})$. For example, $S(\mathfrak{M}(C-T, C-$ $B, C-4)$ ) (the system of $\mathfrak{M}(C-T, C-B, C-4)$ ) is the class of sentences (in our language) that are valid in the class of models that satisfy the conditions $C-T, C-B$ and $C-4$.

There are many interesting relationships between the conditions in Figure 1. Most of them are well-known and require no further comments (see Chellas 1980, ch. 4-5 for more on the relationships between some normal modal systems). However, there are some interesting connections between $C-F$ and some other conditions that are worth mentioning.

$C-D$ says that every possible world can see at least one possible world, and $C-F$ says that every possible world can see at most one possible world. Hence, $C-D \forall x \exists y \Re x y$ and $C-F \forall x \forall y \forall z((\Re x y \wedge \Re x z) \rightarrow y=z)$ entail the following condition: $\forall x \exists y(\Re x y \wedge \forall z(\Re x z \rightarrow y=z))$, which says that every possible world can see exactly one possible world. Hence, every world can see one and exactly one possible world if we assume $C-D$ and $C-F$. In other words, $C-D$ and $C-F$ entail that $\mathfrak{R}$ is a function (that takes one possible world as input and gives one possible world as output). If a model satisfies $C-F$, then $\diamond P \rightarrow \square P$ and $\square P \vee \square \neg P$ are valid in this model, and if a model satisfies $C-D$, then $\square P \rightarrow \diamond P$ is valid in this model. Hence, if a model satisfies both $C-D$ and $C-F, \diamond P \leftrightarrow \square P, \diamond \neg P \leftrightarrow \neg \diamond P$ and - $P \leftrightarrow P$ are valid in this model. Then, the distinctions between possibility and necessity and between non-necessity and impossibility collapse. 
$C-T$ says that $\mathfrak{R}$ is reflexive, that is, that every possible world can see itself, and $C-F$ says that every possible world can see at most one possible world. Hence, $C-T \forall x \mathfrak{R} x x$ and $C-F \forall x \forall y \forall z((\mathfrak{R} x y \wedge \mathfrak{R} x z) \rightarrow y=z)$ entail the following condition $\forall x(\Re x x \wedge \forall y(\Re x y \rightarrow y=x))$, which says that every possible world can see itself and nothing but itself. Furthermore, if a model satisfies $\forall x(\Re x x \wedge \forall y(\Re x y \rightarrow y=x))$, then it also satisfies the following condition $\forall x \forall y(\Re x y \leftrightarrow x=y)$. In other words, if every world can see itself and nothing but itself, then a possible world $y$ is accessible from a possible world $x$ iff $x$ is identical to $y$. So, if a model satisfies $C-T$ and $C-F$, then $P \leftrightarrow \square P, \neg P \leftrightarrow \neg \diamond P$ and $\neg P \leftrightarrow \diamond P$ are valid in this model. Hence, the distinctions between truth and necessary truth and between falsehood and impossibility collapse. But if $C-T$ holds, then $C-D$ also holds. So, it is also true that if a model satisfies $C-T$ and $C-F$, then $\diamond P \leftrightarrow \square P$, $\diamond \neg P \leftrightarrow \neg \diamond P$ and $\mathbf{\square} P \leftrightarrow \diamond P$ are valid in this model. It follows that the distinctions between what is true, possible and necessary collapse. That is, the following equivalences hold $P \leftrightarrow \diamond P \leftrightarrow \square P$. Likewise, we have $\neg P \leftrightarrow \diamond \neg P \leftrightarrow \neg \diamond P \leftrightarrow \mathbf{Q} P \leftrightarrow \diamond P$. The distinctions between what is false, possibly false, impossible and unnecessary also collapse.

\section{Proof theory}

In this section, I will develop a set of tableau systems. The propositional part of these systems is similar to systems introduced by (Smullyan 1968) and (Jeffrey 1967), and the modal part is similar to systems discussed by (Priest 2008). The new interesting thing is the rules for the propositional quantifiers. For more information about the tableau method and various kinds of tableau systems, see, for example, (D’Agostino, Gabbay, Hähnle and Posegga 1999) and (Fitting and Mendelsohn 1998). 


\subsection{Tableau rules}

\subsubsection{Propositional rules}

\begin{tabular}{c|c|c}
\hline$\neg \neg$ & $\wedge$ & $\neg \wedge$ \\
\hline$\neg \neg A, i$ & $A \wedge B, i$ & $\neg(A \wedge B), i$ \\
$\downarrow$ & $\downarrow$ & $\swarrow \searrow$ \\
$A, i$ & $A, i$ & $\neg A, i \neg B, i$ \\
& $B, i$ & \\
\hline$\vee$ & $\neg \vee$ & $\rightarrow$ \\
\hline$A \vee B, i$ & $\neg(A \vee B), i$ & $A \rightarrow B, i$ \\
$\swarrow \searrow$ & $\downarrow$ & $\swarrow \searrow$ \\
$A, i B, i$ & $\neg A, i$ & $\neg A, i \quad B, i$ \\
$\neg \rightarrow$ & $\neg B, i$ & $\neg \leftrightarrow$ \\
\hline$\neg(A \rightarrow B), i$ & $A \leftrightarrow B, i$ & $\neg(A \leftrightarrow B), i$ \\
$\downarrow$ & $\swarrow \searrow$ & $\swarrow \searrow$ \\
$A, i$ & $A, i \neg A, i$ & $A, i \neg A, i$ \\
$\neg B, i$ & $B, i \neg B, i$ & $\neg B, i \quad B, i$ \\
\hline
\end{tabular}

Figure 2: Propositional rules

\subsubsection{Basic modal rules}

\begin{tabular}{c|c|c|c}
\hline$\square$ & $\diamond$ & $\neg \square$ & $\neg \diamond$ \\
\hline$\square A, i$ & $\diamond A, i$ & $\neg \square A, i$ & $\neg \diamond A, i$ \\
$i r j$ & $\downarrow$ & $\downarrow$ & $\downarrow$ \\
$\downarrow$ & $i r j$ & $\diamond \neg A, i$ & $\square \neg A, i$ \\
$A, j$ & $A, j$ & & \\
\hline
\end{tabular}

Figure 3: Basic modal rules 


\subsubsection{Quantifier rules}

\begin{tabular}{c|c|c|c}
\hline$\forall$ & $\exists$ & $\neg \forall$ & $\neg \exists$ \\
\hline$\forall X A, i$ & $\exists X A, i$ & $\neg \forall X A, i$ & $\neg \exists X A, i$ \\
$\downarrow$ & $\downarrow$ & $\downarrow$ & $\downarrow$ \\
$A[B / X], i$ & $A[Y / X], i$ & $\exists X \neg A, i$ & $\forall X \neg A, i$ \\
\hline
\end{tabular}

Figure 4: Propositional quantifier rules

Note that in $(\forall), B$ is any (quantifier-free) formula (in $\mathcal{L}$ ) that is substitutable for $X$ in $A$; and in $(\exists), Y$ is a propositional variable that is new to the branch.

\subsubsection{Accessibility rules}

\begin{tabular}{c|c|c|c|c|c}
\hline$T-D$ & $T-T$ & $T-B$ & $T-F$ & $T-4$ & $T-5$ \\
\hline$i$ & $i$ & $i r j$ & $i r j$ & $i r j$ & $i r j$ \\
$\downarrow$ & $\downarrow$ & $\downarrow$ & $i r k$ & $j r k$ & $i r k$ \\
$i r j$ & $i r i$ & $j r i$ & $\downarrow$ & $\downarrow$ & $\downarrow$ \\
where $j$ is new & & & $j=k$ & $i r k$ & $j r k$ \\
\hline
\end{tabular}

Figure 5: Accessibility rules

\subsubsection{The CUT-rule and the identity-rules}

\begin{tabular}{c|c|c}
\hline$C U T$ & $T-I i$ & $T-I i i$ \\
\hline$*$ & $A(i)$ & $A(j)$ \\
$\swarrow \searrow$ & $i=j$ & $i=j$ \\
$A, i \neg A, i$ & $\downarrow$ & $\downarrow$ \\
for every $A$ and $i$ & $A(j)$ & $A(i)$ \\
\hline
\end{tabular}

Figure 6: The CUT-rule and the identity-rules

The identity rules should be interpreted in the following way. $A(i)$ is a line in a tableau that includes ' $i$ ', and $A(j)$ is like $A(i)$ except that ' $i$ ' is replaced by 
' $j$ '. That is, if $A(i)$ is $A, i$, then $A(j)$ is $A, j$; if $A(i)$ is $k r i$, then $A(j)$ is $k r j$; if $A(i)$ is $i=k$, then $A(j)$ is $j=k$, etc. ' $*$ ' in $C U T$ indicates that $C U T$ is a rule without any 'premises'.

\subsection{Tableau systems and some basic proof-theoretical concepts}

A tableau system is a set of tableau rules. A normal modal tableau system is a tableau system that includes all propositional rules and all basic modal rules. The smallest normal modal tableau system without any accessibility rules is called $K$. By adding various additional accessibility rules, we obtain a large class of stronger systems. By combining the different rules in various ways, we can construct 64 different normal modal tableau systems (many of these are deductively equivalent, that is, include the same theorems). To make some proofs easier, it is often useful to add the $C U T$-rule. The identity rules are included in every system that contains $T-F$. If $S$ is a normal modal system, then $S_{E x t}$ is the normal modal system $S$ extended by the quantifier rules. There are 64 extended systems of this kind. All in all, we consider 128 different systems in this paper. Many of these are deductively equivalent, that is, contain the same theorems.

Let $T_{1}, \ldots, T_{n}$ be the (normal modal) tableau system that includes the tableau rules $T_{1}, \ldots, T_{n}$. The initial $T$ may be omitted if it is clear that we are talking about a tableau system. Then, TB4 is the (normal modal) tableau system that includes the tableau rules $T-T, T-B$ and $T-4$, etc.

The concepts of semantic tableau, branch, open and closed branch, etc. are essentially defined as usual. A (semantic) tableau is a tree-like structure where the nodes in the tree have the following form: $A, i$, where $A$ is a formula in our language and $i$ is in $\{0,1,2,3, \ldots\}$, or something of the form $i r j$, or $i=j$ where $i, j$ is in $\{0,1,2,3, \ldots\}$. A branch in a tableau is closed iff there is a formula $A$ and a number $i$, such that both $A, i$ and $\neg A, i$ occur on the branch or if we have $\neg \top, i$ or $\perp, i$ on the branch; it is open just in case it is not closed. A tableau itself is closed iff every branch in it is closed; it is open iff it is not closed.

Let $S$ be any system in this paper and let an $S$-tableau be a tableau generated in accordance with the rules in $S$. Furthermore, let $A$ be a sentence and let $\Gamma$ be a finite set of sentences. ' $\vdash$ S $A$ ' says that $A$ is a theorem in $S$ and 
' $\Gamma \vdash_{S} A$ ' says that $A$ is derivable from $\Gamma$ in $S$.

Proof in a system. A proof of $A$ in $S$ is a closed $S$-tableau that starts with $\neg A, 0$.

Theorem in a system. A sentence $A$ is a theorem in $S$ or provable in $S, \vdash_{S} A$, iff there is a proof of $A$ in $S$, that is, iff there is a closed $S$-tableau that starts with $\neg A, 0$.

Derivation in a system. A derivation in the system $S$ of the sentence $A$ from the set of sentences $\Gamma$, is a closed $S$-tableau whose initial list comprises $B, 0$ for every $B$ in $\Gamma$ and $\neg A, 0$. The sentences in $\Gamma$ are the premises of the derivation and $A$ is called the conclusion of the derivation. The initial list of a tableau consists of the first nodes in this tableau whose 'satisfiability' we are testing.

Proof-theoretic consequence in a system. A sentence $A$ is a prooftheoretic consequence of a set of sentences $\Gamma$ in $S$ or $A$ is derivable from $\Gamma$ in $S, \Gamma \vdash_{S} A$, iff there is a derivation of $A$ in $S$ from $\Gamma$, that is, iff there is a closed $S$-tableau whose initial list comprises $B, 0$ for every $B$ in $\Gamma$ and $\neg A, 0$.

\subsection{Some theorems}

We are now in a position to prove some propositions. I will go through some theorems that appear in (Lewis and Langford 1932, 178-198) to illustrate how to use our systems and especially the new quantifier rules. I will also consider some additional theorems that are not discussed by Lewis and Langford.

First, let us mention some important relationships between some fundamental modal concepts. All of the following sentences are theorems in every system in this paper (some hold by definition): $\diamond P \leftrightarrow(P \circ P), \diamond P \leftrightarrow$ $\neg(P \circ P), \square P \leftrightarrow \neg(\neg P \circ \neg P), \mathbf{\square} P \leftrightarrow(\neg P \circ \neg P),(P \bullet Q) \leftrightarrow \neg(P \circ Q)$, $(P \Rightarrow Q) \leftrightarrow \neg(P \circ \neg Q),(P \Leftrightarrow Q) \leftrightarrow(\neg(P \circ \neg Q) \wedge \neg(Q \circ \neg P)), \diamond P \leftrightarrow$ $\neg(P \bullet P), P \leftrightarrow(P \bullet P), \square P \leftrightarrow(\neg P \bullet \neg P), \boldsymbol{\square} P \leftrightarrow \neg(\neg P \bullet \neg P),(P \circ Q) \leftrightarrow$ $\neg(P \bullet Q),(P \Rightarrow Q) \leftrightarrow(P \bullet \neg Q),(P \Leftrightarrow Q) \leftrightarrow((P \bullet \neg Q) \wedge(Q \bullet \neg P))$, $\diamond P \leftrightarrow \neg(P \Rightarrow \neg P), \diamond P \leftrightarrow(P \Rightarrow \neg P), \square P \leftrightarrow(\neg P \Rightarrow P), \mathbf{\square} P \leftrightarrow$ $\neg(\neg P \Rightarrow P),(P \circ Q) \leftrightarrow \neg(P \Rightarrow \neg Q),(P \bullet Q) \leftrightarrow(P \Rightarrow \neg Q), \quad(P \Leftrightarrow$ $Q) \leftrightarrow((P \Rightarrow Q) \wedge(Q \Rightarrow P)), \diamond P \leftrightarrow \neg \square \neg P, \diamond P \leftrightarrow \square \neg P, \mathbf{\square} P \leftrightarrow \neg \square P$, 


$$
\begin{aligned}
& (P \circ Q) \leftrightarrow \neg \square \neg(P \wedge Q),(P \bullet Q) \leftrightarrow \square \neg(P \wedge Q),(P \Rightarrow Q) \leftrightarrow \square(P \rightarrow Q), \\
& (P \Leftrightarrow Q) \leftrightarrow \square(P \leftrightarrow Q), \diamond P \leftrightarrow \neg \diamond P, \square P \leftrightarrow \neg \diamond \neg P, \square P \leftrightarrow \diamond \neg P, \\
& (P \circ Q) \leftrightarrow \diamond(P \wedge Q),(P \bullet Q) \leftrightarrow \neg \diamond(P \wedge Q),(P \Rightarrow Q) \leftrightarrow \neg \diamond \neg(P \rightarrow Q), \\
& (P \Leftrightarrow Q) \leftrightarrow \neg \diamond \neg(P \leftrightarrow Q), \diamond P \leftrightarrow \neg \diamond P, \square P \leftrightarrow \bullet \neg P, \square P \leftrightarrow \neg \diamond \neg P, \\
& (P \circ Q) \leftrightarrow \neg \diamond(P \wedge Q),(P \bullet Q) \leftrightarrow \diamond(P \wedge Q),(P \Rightarrow Q) \leftrightarrow \diamond \neg(P \rightarrow Q), \\
& (P \Leftrightarrow Q) \leftrightarrow \diamond \neg(P \leftrightarrow Q) .
\end{aligned}
$$

Lewis observes that we can prove that there exists at least one proposition which is true and that there exists at least one false proposition. We can also show that not everything is true $(\neg \forall X X)$ and that not everything is false $(\neg \forall X \neg X)$. Let us verify the claim that there is something true and that there is something false.

$$
\begin{gathered}
\exists X X \text {. Something is true. } \\
\text { (1) } \neg \exists X X, 0 \\
(2) \forall X \neg X, 0[1, \neg \exists] \\
(3) \neg(P \vee \neg P), 0[2, \forall(\neg X)[P \vee \neg P / X]] \\
(4) \neg P, 0[3, \neg \vee] \\
(5) \neg \neg P, 0[3, \neg \vee] \\
(6) *[4,5] \\
\exists X \neg X \text {. Something is false. } \\
(1) \neg \exists X \neg X, 0 \\
(2) \forall X \neg \neg X, 0[1, \neg \exists] \\
(3) \neg \neg(P \wedge P), 0[2, \forall(\neg \neg X)[P \wedge \neg P / X]] \\
(4) P \wedge \neg P, 0[3, \neg \neg] \\
(5) P, 0[4, \wedge] \\
(6) \neg P, 0[4, \wedge] \\
(7) *[5,6]
\end{gathered}
$$

Here are some other theorems that do not contain any modal operators. All of these sentences can be proved in all our systems. $\forall X(\perp \rightarrow X)$ : Falsum (a contradiction) materially implies anything. $\forall X(X \rightarrow \top)$ : Everything materially implies Verum (something that is logically true). $\forall X(X \rightarrow$ $\forall Y(Y \rightarrow X))$ : If something is true it is materially implied by anything. $\forall X(\forall Y(Y \rightarrow X) \rightarrow X)$ : If something is materially implied by anything, it is true. $\forall X(X \leftrightarrow \forall Y(Y \rightarrow X))$ : Something is true iff it is materially implied by anything. $\forall X(\neg X \rightarrow \forall Y(X \rightarrow Y))$ : If something is false, it materially implies everything. $\forall X(\forall Y(X \rightarrow Y) \rightarrow \neg X)$ : If something materially implies everything, it is false. $\forall X(\neg X \leftrightarrow \forall Y(X \rightarrow Y))$ : Something is false iff it materially implies everything. 
All of the following sentences are theorems in every system in this paper:

$\forall X(\exists Y((Y \rightarrow X) \wedge(\neg Y \rightarrow X)) \rightarrow X), \forall X(X \rightarrow \exists Y((Y \rightarrow X) \wedge(\neg Y \rightarrow$ $X))), \forall X(X \leftrightarrow \exists Y((Y \rightarrow X) \wedge(\neg Y \rightarrow X)))$ (X is true iff there is a $Y$ such that both $Y$ and the negation of $Y$ implies $X), \forall X(\exists Y((X \rightarrow Y) \wedge(X \rightarrow$ $\neg Y)) \rightarrow \neg X), \forall X(\neg X \rightarrow \exists Y((X \rightarrow Y) \wedge(X \rightarrow \neg Y))), \forall X(\neg X \leftrightarrow \exists Y((X \rightarrow$ $Y) \wedge(X \rightarrow \neg Y)))(X$ is false iff there is a $Y$ such that $X$ implies both $Y$ and the negation of $Y)$.

Let $A$ be a formula that does not contain any free occurrence of $X$. Then all equivalences of the following forms are provable: $\forall X(X \rightarrow A) \leftrightarrow(\exists X X \rightarrow$ $A), \forall X(A \rightarrow X) \leftrightarrow(A \rightarrow \forall X X), \exists X(A \rightarrow X) \leftrightarrow(A \rightarrow \exists X X)$. I will show the first and leave the rest to the reader.

$$
\begin{aligned}
& \forall X(X \rightarrow A) \leftrightarrow(\exists X X \rightarrow A) \\
& \text { Left to right } \forall X(X \rightarrow A) \rightarrow(\exists X X \rightarrow A) \\
& \text { (1) } \neg(\forall X(X \rightarrow A) \rightarrow(\exists X X \rightarrow A)), 0 \\
& \text { (2) } \forall X(X \rightarrow A), 0[1, \neg \rightarrow] \\
& \text { (3) } \neg(\exists X X \rightarrow A), 0[1, \neg \rightarrow] \\
& \text { (4) } \exists X X, 0[3, \neg \rightarrow] \\
& \text { (5) } \neg A, 0[3, \neg \rightarrow] \\
& \text { (6) } Y, 0[4, \exists(X)[Y / X]] \\
& \text { (7) } Y \rightarrow A, 0[2, \forall(X \rightarrow A)[Y / X]] \\
& \begin{array}{cc}
\swarrow & \searrow \\
\text { (8) } \neg Y, 0[7, \rightarrow] & (9) A, 0[7, \rightarrow]
\end{array} \\
& (10) *[6,8] \quad(11) *[5,9]
\end{aligned}
$$

$(13) *[6,12]$

Lewis proves that there are at least two propositions that are distinct (not necessarily equivalent). We cannot prove this claim in every system in this paper. However, we can show that it holds in every system that includes $T-T$ 
(or $T-D) . \exists X \exists Y \neg(X \Leftrightarrow Y)$ is by definition equivalent with $\exists X \exists Y \neg \square(X \leftrightarrow$ $Y)$. So, to prove the former, we show the latter.

$\exists X \exists Y \neg \square(X \leftrightarrow Y)$. There are at least two propositions that are not necessarily equivalent.

(1) $\neg \exists X \exists Y \neg \square(X \leftrightarrow Y), 0$

(2) $\forall X \neg \exists Y \neg \square(X \leftrightarrow Y), 0[1, \neg \exists]$

(3) $\neg \exists Y \neg \square(\top \leftrightarrow Y), 0[2, \forall(\neg \exists Y \neg \square(X \leftrightarrow Y))[\top / X]]$

(4) $\forall Y \neg \neg \square(\top \leftrightarrow Y), 0[3, \neg \exists]$

(5) $\neg \neg \square(T \leftrightarrow \perp), 0[4, \forall(\neg \neg \square(T \leftrightarrow Y))[\perp / Y]]$

(6) $\square(\top \leftrightarrow \perp), 0[5, \neg \neg]$

(7) $0 \mathrm{r} 0[T]$

(8) $\top \leftrightarrow \perp, 0[6,7, \square]$

(9) $\top, 0[8, \leftrightarrow]$

(11) $\perp, 0[8, \leftrightarrow]$

$(13) *[11]$

(10) $\neg \top, 0[8, \leftrightarrow]$

$(12) \neg \perp, 0[8, \leftrightarrow]$

$(14) *[10]$

This proof depends on $T$ at Step 7. This step is essential (however, in systems that include $D$ it can be replaced by ' $0 r 1$ ' if (8)-(12) are modified in an obvious way). If a possible world cannot see any world, then everything is necessary in this world. Hence, all equivalences are necessary in this world.

In every system in this paper, we can show that any two necessary propositions are necessarily equivalent and hence not distinct, $\forall X \forall Y((\square X \wedge \square Y) \rightarrow$ $(X \Leftrightarrow Y))$, and that any two impossible propositions are necessarily equivalent and hence not distinct, $\forall X \forall Y((\diamond X \wedge Y) \rightarrow(X \Leftrightarrow Y))$. It is left to the reader to verify this claim.

We have seen that there is something true and that there is something false. We can also show that some propositions are necessarily true $(\exists X \square X)$, that some propositions are possibly true $(\exists X \diamond X)$, that some propositions are necessarily false $(\exists X \square \neg X)$, that some propositions cannot be true $(\exists X \neg \diamond X)$, that some propositions are possibly false $(\exists X \diamond \neg X)$, that some propositions are not necessarily true $(\exists X \neg \square X)$, that some propositions are not necessarily false $(\exists X \neg \square \neg X)$, and that some propositions cannot be false $(\exists X \neg \diamond \neg X)$. The proof of $\exists X \diamond X$ requires $T$. It can also be proved in systems with $D$. If a possible world cannot see any possible world, then nothing is possible in this world. Then we cannot conclude that there is something possible in this world. Similar things can be said about $\exists X \diamond \neg X, \exists X \neg \square X$ and $\exists X \neg \square \neg X$. All the other propositions hold in every system. I will establish that there are necessary truths and leave the other proofs to the reader.

Organon F 26 (3) 2019: 505-536 
$\exists X \square X$. Some propositions are necessarily true. There are necessary truths.

(1) $\neg \exists X \square X, 0$

(2) $\forall X \neg \square X, 0[1, \neg \exists]$

(3) $\neg \square(P \leftrightarrow P), 0[2, \forall(\neg \square X)[P \leftrightarrow P / X]]$

$(4) \diamond \neg(P \leftrightarrow P), 0[3, \neg \square]$

(5) $0 r 1[4, \diamond]$

(6) $\neg(P \leftrightarrow P), 1[4, \diamond]$

(7) $P, 1[6, \neg \leftrightarrow]$

(9) $\neg P, 1[6, \neg \leftrightarrow]$

$(11) *[7,9]$

(8) $\neg P, 1[6, \neg \leftrightarrow]$

(10) $P, 1[6, \neg \leftrightarrow]$

$(12) *[8,10]$

Lewis observes that no proposition is equivalent to its own negation. We can prove this proposition in every system that includes $T$ (or $D) . \forall X \neg(X \Leftrightarrow$ $\neg X)$ is by definition equivalent with $\forall X \neg \square(X \leftrightarrow \neg X)$.

In our systems, we can prove several Barcan-like formulas. All of the following sentences are provable in every system in this paper: if everything is necessarily true, then it is necessary that everything is true $(\forall X \square X \rightarrow \square \forall X X)$, if it is necessary that everything is true, then everything is necessarily true $(\square \forall X X \rightarrow \forall X \square X)$, it is necessary that everything is true iff everything is necessarily true $(\square \forall X X \leftrightarrow \forall X \square X)$, if something is possibly true, then it is possible that something is true $(\exists X \diamond X \rightarrow \diamond \exists X X)$, if it is possible that something is true, then something is possibly true $(\diamond \exists X X \rightarrow \exists X \diamond X)$, it is possible that something is true iff something is possibly true $(\diamond \exists X X \leftrightarrow \exists X \diamond X)$, if something is necessarily true, then it is necessary that something is true ( $\exists X \square X \rightarrow \square \exists X X$ ), if it is possible that everything is true, then everything is possibly true $(\diamond \forall X X \rightarrow \forall X \diamond X)$. I will prove the first and leave the rest to the reader.

$\forall X \square X \rightarrow \square \forall X X$. If everything is necessarily true, then it is necessary that everything is true.

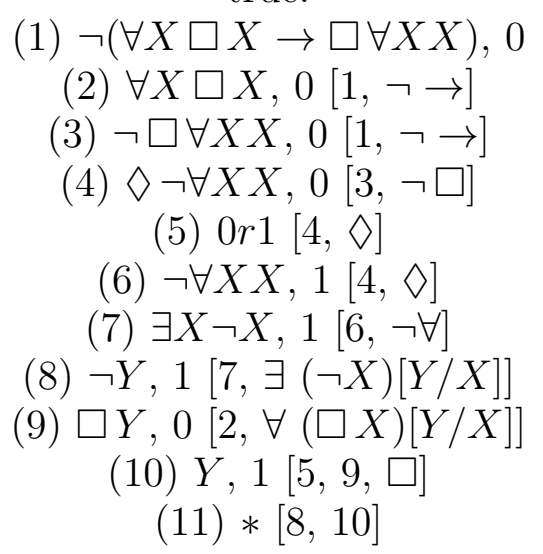

Organon F 26 (3) 2019: 505-536 
Here are some other theorems that can be proved in our systems: $\exists Y(P \wedge$ $Y) \rightarrow P, P \leftrightarrow \exists Y(P \wedge Y), \neg P \rightarrow \forall Y \neg(P \wedge Y), \exists Y((P \rightarrow Y) \wedge(Y \rightarrow$ $R)) \rightarrow(P \rightarrow R), P \rightarrow \forall Y(Y \rightarrow P), P \leftrightarrow \forall Y(Y \rightarrow P), \neg P \rightarrow \forall Y(P \rightarrow Y)$, $\neg P \leftrightarrow \forall Y(P \rightarrow Y), \neg \diamond P \rightarrow \exists Y(\square(P \rightarrow Y) \wedge \square(P \rightarrow \neg Y)), \exists Y(\square(P \rightarrow$ $Y) \wedge \square(P \rightarrow \neg Y)) \rightarrow \neg \diamond P, \diamond P \rightarrow \forall Y(\neg \square(P \rightarrow Y) \vee \neg \square(P \rightarrow \neg Y))$, $\forall Y(\neg \square(P \rightarrow Y) \vee \neg \square(P \rightarrow \neg Y)) \rightarrow \diamond P, \exists Y(\square(Y \rightarrow P) \wedge \square(\neg Y \rightarrow P)) \rightarrow$ $\square P, \square P \rightarrow \exists Y(\square(Y \rightarrow P) \wedge \square(\neg Y \rightarrow P))$.

Now, let us consider some theorems that include the consistency operator (see Lewis and Langford, p. 196). $\exists X((X \Rightarrow Q) \wedge(X \circ R)) \rightarrow(Q \circ R)$ : If there is an $X$ such that $X$ necessarily implies $Q$ and $X$ is consistent with $R$, then $Q$ is consistent with $R . \exists Y((P \Rightarrow Y) \wedge \neg(Y \circ R)) \rightarrow \neg(P \circ R)$ : If there is a $Y$ such that $P$ necessarily implies $Y$ and $Y$ is inconsistent with $R$, then $P$ is inconsistent with $R . \exists X \exists Y((X \Rightarrow R) \wedge(Y \Rightarrow S) \wedge(X \circ Y)) \rightarrow$ $(R \circ S)$ : If there is an $X$ and a $Y$ such that $X$ necessarily implies $R$ and $Y$ necessarily implies $S$ and $X$ is consistent with $Y$, then $R$ is consistent with $S$. $\exists Y((P \Rightarrow Y) \wedge(P \Rightarrow \neg Y)) \rightarrow \neg(P \circ P), \exists X((X \Rightarrow Q) \wedge(X \circ X)) \rightarrow(Q \circ Q)$, $\exists Y((P \Rightarrow Y) \wedge \neg(Y \circ Y)) \rightarrow \neg(P \circ P),(P \circ P) \rightarrow \forall Y \neg((P \Rightarrow Y) \wedge(P \Rightarrow$ $\neg Y)),(P \circ P) \rightarrow \forall Y((P \circ Y) \vee(P \circ \neg Y)), \exists X \exists Y((P \Rightarrow X) \wedge(Q \Rightarrow Y) \wedge$ $\neg(X \circ Y)) \rightarrow \neg(P \circ Q)$ : If there is an $X$ and a $Y$ such that $P$ necessarily implies $X$ and $Q$ necessarily implies $Y$ and $X$ is inconsistent with $Y$, then $P$ is inconsistent with $Q$. By definition the last formula is equivalent with the following sentence: $\exists X \exists Y(\square(P \rightarrow X) \wedge \square(Q \rightarrow Y) \wedge \neg \diamond(X \wedge Y)) \rightarrow$ $\neg \diamond(P \wedge Q)$. So, to prove the former, it is sufficient to establish the latter. Let us prove this formula (' $M P$ ' is an abbreviation of 'Modus Ponens', which is a derived rule in our systems).

$$
\begin{gathered}
\exists X \exists Y(\square(P \rightarrow X) \wedge \square(Q \rightarrow Y) \wedge \neg \diamond(X \wedge Y)) \rightarrow \neg \diamond(P \wedge Q) . \\
\neg(\exists X \exists Y((\square(P \rightarrow X) \wedge \square(Q \rightarrow Y)) \wedge \neg \diamond(X \wedge Y)) \rightarrow \neg \diamond(P \wedge Q)) \\
(2) \exists X \exists Y((\square(P \rightarrow X) \wedge \square(Q \rightarrow Y)) \wedge \neg \diamond(X \wedge Y)), 0[1, \neg \rightarrow] \\
(3) \neg \neg \diamond(P \wedge Q), 0[1, \neg \rightarrow] \\
(4) \diamond(P \wedge Q), 0[3, \neg]] \\
(5) \exists Y((\square(P \rightarrow Z) \wedge \square(Q \rightarrow Y)) \wedge \neg \diamond(Z \wedge Y)), 0[2, \exists] \\
(6)(\square(P \rightarrow Z) \wedge \square(Q \rightarrow W)) \wedge \neg \diamond(Z \wedge W), 0[5, \exists] \\
(7) \square(P \rightarrow Z) \wedge \square(Q \rightarrow W), 0[6, \wedge] \\
(8) \neg \diamond(Z \wedge W), 0[6, \wedge] \\
(9) \square(P \rightarrow Z), 0[7, \wedge] \\
(10) \square(Q \rightarrow W), 0[7, \wedge] \\
(11) \square \neg(Z \wedge W), 0[8, \neg \diamond] \\
(12) 0 r 1[4, \diamond] \\
(13) P \wedge Q, 1[4, \diamond]
\end{gathered}
$$$$
\text { (1) } \neg(\exists X \exists Y((\square(P \rightarrow X) \wedge \square(Q \rightarrow Y)) \wedge \neg \diamond(X \wedge Y)) \rightarrow \neg \diamond(P \wedge Q)), 0
$$$$
\text { (5) } \exists Y((\square(P \rightarrow Z) \wedge \square(Q \rightarrow Y)) \wedge \neg \diamond(Z \wedge Y)), 0[2, \exists]
$$

Organon F 26 (3) 2019: 505-536 


$$
\begin{aligned}
& \text { (14) } P, 1[13, \wedge] \\
& \text { (15) } Q, 1[13, \wedge] \\
& \text { (16) } P \rightarrow Z, 1[9,12 \text {, } \\
& \text { (17) } Q \rightarrow W, 1[10,12, \square] \\
& \text { (18) } Z, 1[14,16, M P] \\
& \text { (19) } W, 1[15,17, M P] \\
& (20) \neg(Z \wedge W), 1[11,12, \square] \\
& \text { (21) } \neg Z, 1[20, \neg \wedge] \\
& (23) *[18,21] \quad(24) *[19,22]
\end{aligned}
$$

Here are some other interesting theorems that can be proved in our systems.

$\forall X(\square X \rightarrow \forall Y \square(Y \rightarrow X))$ : If something is necessarily true it is necessarily implied by anything. $\forall X(\forall Y \square(Y \rightarrow X) \rightarrow \square X)$ : If something is necessarily implied by anything, it is necessarily true. $\forall X(\square X \leftrightarrow \forall Y \square(Y \rightarrow$ $X)$ ): Something is necessarily true iff it is necessarily implied by anything. $\forall X(\ \rightarrow \forall Y \square(X \rightarrow Y))$ : If something is impossible, it necessarily implies everything. $\forall X(\forall Y \square(X \rightarrow Y) \rightarrow \diamond X)$ : If something necessarily implies everything, it is impossible. $\forall X(\diamond X \leftrightarrow \forall Y \square(X \rightarrow Y))$ : Something is impossible iff it necessarily implies everything. $\forall X(\diamond X \rightarrow \neg \forall Y \square(X \rightarrow Y))$ : If something is possible, it is not the case that it necessarily implies everything. $\forall X(\neg \forall Y \square(X \rightarrow Y) \rightarrow \diamond X)$ : If it is not the case that something necessarily implies everything, then it is possible. $\forall X(\diamond X \leftrightarrow \neg \forall Y \square(X \rightarrow Y))$ : $X$ is possible iff it is not the case that $X$ necessarily implies everything. $\forall X(\square X \leftrightarrow \forall Y \square(\neg X \rightarrow Y))$ : It is necessary that $X$ iff the negation of $X$ implies everything. $\forall X(\diamond X \leftrightarrow \exists Y \neg \square(X \rightarrow Y)): X$ is possible iff there is something $X$ does not necessarily imply. $\forall X(\diamond X \leftrightarrow \neg \exists Y \neg \square(X \rightarrow Y)): X$ is impossible iff there is nothing $X$ does not necessarily imply. $\forall X(\square X \leftrightarrow$ $\neg \exists Y \neg \square(\neg X \rightarrow Y)): X$ is necessary iff there is nothing that the negation of $X$ does not imply. The theorems in this paragraph tell us something about the necessary and sufficient conditions for something to be necessary, possible and impossible. Our next theorems also consider such conditions.

$\forall X(\exists Y(\square(Y \rightarrow X) \wedge \square(\neg Y \rightarrow X)) \rightarrow \square X):$ If there is a proposition such that both it and its negation necessarily implies $X$, then $X$ is necessary. $\forall X(\square X \rightarrow \exists Y(\square(Y \rightarrow X) \wedge \square(\neg Y \rightarrow X)))$ : If $X$ is necessary, then there is a proposition such that both it and its negation necessarily implies $X . \forall X(\square X \leftrightarrow \exists Y(\square(Y \rightarrow X) \wedge \square(\neg Y \rightarrow X)))$ : Something $X$ is necessary iff there is a proposition such that both it and its negation necessarily 
implies $X . \forall X(\exists Y(\square(X \rightarrow Y) \wedge \square(X \rightarrow \neg Y)) \rightarrow X)$ : If there is a proposition such that $X$ necessarily implies both it and its negation, then $X$ is impossible. $\forall X(\diamond X \rightarrow \exists Y(\square(X \rightarrow Y) \wedge \square(X \rightarrow \neg Y)))$ : If $X$ is impossible, then there is a proposition such that $X$ necessarily implies both it and its negation. $\forall X(\diamond X \leftrightarrow \exists Y(\square(X \rightarrow Y) \wedge \square(X \rightarrow \neg Y)))$ : $X$ is impossible iff there is a proposition such that $X$ necessarily implies both it and its negation. $\forall X(\neg \exists Y(\square(X \rightarrow Y) \wedge \square(X \rightarrow \neg Y)) \rightarrow \diamond X)$ : If there is no proposition such that $X$ necessarily implies both it and its negation, then $X$ is possible. $\forall X(\diamond X \rightarrow \neg \exists Y(\square(X \rightarrow Y) \wedge \square(X \rightarrow \neg Y)))$ : If $X$ is possible, then there is no proposition such that $X$ necessarily implies both it and its negation. $\forall X(\diamond X \leftrightarrow \neg \exists Y(\square(X \rightarrow Y) \wedge \square(X \rightarrow \neg Y)))$ : $X$ is possible iff there is no proposition such that $X$ necessarily implies both it and its negation. $\forall X(\square X \leftrightarrow \forall Y(\square(Y \rightarrow X) \wedge \square(\neg Y \rightarrow X))), \forall X(\diamond X \leftrightarrow \neg \forall Y(\square(X \rightarrow$ $Y) \wedge \square(X \rightarrow \neg Y))), \forall X(\diamond X \leftrightarrow \forall Y(\neg \square(X \rightarrow Y) \vee \neg \square(X \rightarrow \neg Y)))$, $\forall X(\diamond X \leftrightarrow \exists Y(\neg \square(X \rightarrow Y) \vee \neg \square(X \rightarrow \neg Y))), \forall X(\diamond X \leftrightarrow \forall Y(\square(X \rightarrow$ $Y) \wedge \square(X \rightarrow \neg Y)))$.

We can prove the following sentences in our systems: $\forall X(\exists Y(\square(X \rightarrow$ $Y) \wedge \square(X \rightarrow \neg Y)) \leftrightarrow \forall Y(\square(X \rightarrow Y) \wedge \square(X \rightarrow \neg Y)))$, there is a proposition such that $X$ necessarily implies both it and its negation iff it is true of every proposition that $X$ necessarily implies both it and its negation. $\forall X(\exists Y(\square(Y \rightarrow X) \wedge \square(\neg Y \rightarrow X)) \leftrightarrow \forall Y(\square(Y \rightarrow X) \wedge \square(\neg Y \rightarrow X)))$, there is a proposition such that both it and its negation implies $X$ iff it is true of every proposition that both it and its negation implies $X$.

In systems with $T$, we can prove the following sentences: $\square \forall X \square X \rightarrow$ $\square \forall X X$ and $\square \forall X \square X \rightarrow \forall X \square X$. In systems with 4 , we can prove the following sentences: $\forall X \square X \rightarrow \square \forall X \square X$ and $\square \forall X X \rightarrow \square \forall X \square X$. Finally, in systems with $T$ and 4 all of the following sentences are equivalent: $\square \forall X X$, $\forall X \square X$ and $\square \forall X \square X$.

Now, let us turn to Lewis's existence postulate.

\subsection{Lewis and Langford's existence postulate}

According to Lewis's so-called existence postulate, which categorically distinguishes strict from material implication, there is some $X$ and $Y$ such that $X$ does not strictly imply $Y$ and $X$ does not strictly imply that $Y$ is false (Lewis and Langford 1932, 179). This postulate can be symbolised in the following way: $\exists X \exists Y(\neg(X \Rightarrow Y) \wedge \neg(X \Rightarrow \neg Y))$, which by definition is equivalent with 
$\exists X \exists Y(\neg \square(X \rightarrow Y) \wedge \neg \square(X \rightarrow \neg Y))$. The existence postulate is equivalent with the proposition that there is something contingent $(\exists X \nabla X)$. Let us now prove this. $\exists X \nabla X$ is, by definition, equivalent with the claim that there is something that is possibly true and possibly false $(\exists X(\diamond X \wedge \diamond \neg X))$. So, to prove that the existence postulate is equivalent with the proposition that there is something contingent, we prove that the existence postulate is equivalent with $\exists X(\diamond X \wedge \diamond \neg X)$. I show one direction and leave the other to the reader.

$\exists X(\diamond X \wedge \diamond \neg X)$ is implied by the existence postulate.

(1) $\neg(\exists X \exists Y(\neg \square(X \rightarrow Y) \wedge \neg \square(X \rightarrow \neg Y)) \rightarrow \exists X(\diamond X \wedge \diamond \neg X)), 0$

(2) $\exists X \exists Y(\neg \square(X \rightarrow Y) \wedge \neg \square(X \rightarrow \neg Y)), 0[1, \neg \rightarrow]$

(3) $\neg \exists X(\diamond X \wedge \diamond \neg X), 0[1, \neg \rightarrow]$

(4) $\forall X \neg(\diamond X \wedge \diamond \neg X), 0[3, \neg \exists]$

(5) $\exists Y(\neg \square(Z \rightarrow Y) \wedge \neg \square(Z \rightarrow \neg Y)), 0[2, \exists Z / X]$

(6) $\neg \square(Z \rightarrow W) \wedge \neg \square(Z \rightarrow \neg W), 0[5, \exists W / Y]$

(7) $\neg \square(Z \rightarrow W), 0[6, \wedge]$

(8) $\neg \square(Z \rightarrow \neg W), 0[6, \wedge]$

(9) $\neg(\diamond(Z \rightarrow W) \wedge \diamond \neg(Z \rightarrow W)), 0[4, \forall(Z \rightarrow W) / X]$

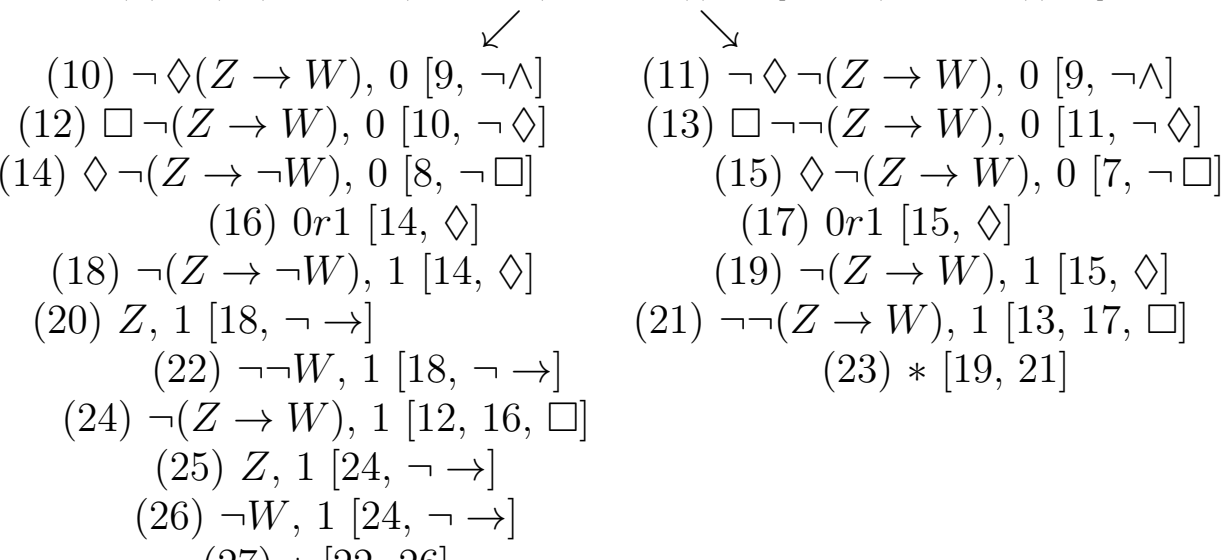

$(27) *[22,26]$

The existence postulate is also equivalent with the proposition that there is an $X$ and a $Y$ such that $X$ is both consistent with $Y$ and with the negation of $Y, \exists X \exists Y((X \circ Y) \wedge(X \circ \neg Y))$, which by definition is equivalent with $\exists X \exists Y(\diamond(X \wedge Y) \wedge \diamond(X \wedge \neg Y))$. All these equivalences hold in every system in this paper.

In all of our systems we can prove the following sentences: if something is contingent, then not everything is non-contingent $(\exists X \nabla X \rightarrow \neg \forall X \boldsymbol{\nabla} X)$, if not everything is non-contingent, then something is contingent $(\neg \forall X \boldsymbol{\nabla} X \rightarrow$ $\exists X \nabla X)$, something is contingent iff not everything is non-contingent $(\exists X \nabla X$ $\leftrightarrow \neg \forall X \boldsymbol{\nabla} X$ ), if everything is contingent, then nothing is non-contingent 
$(\forall X \nabla X \rightarrow \neg \exists X \boldsymbol{\nabla} X)$, if nothing is non-contingent, then everything is contingent $(\neg \exists X \boldsymbol{\nabla} X \rightarrow \forall X \nabla X)$, everything is contingent iff nothing is noncontingent $(\forall X \nabla X \leftrightarrow \neg \exists X \boldsymbol{\nabla} X)$, everything is non-contingent iff nothing is contingent $(\forall X \boldsymbol{\nabla} X \leftrightarrow \neg \exists X \nabla X)$, and something is non-contingent iff not everything is contingent $(\exists X \boldsymbol{\nabla} X \leftrightarrow \neg \forall X \nabla X)$. In every system that includes $T-F$, we can prove that everything is non-contingent, that is, we can show that $\forall X \boldsymbol{\nabla} X$ is valid. $\forall X \boldsymbol{\nabla} X$ is equivalent with the negation of the proposition that there is something contingent. So, $T-F$ is incompatible with the existence postulate. Let us now show that $\forall X \boldsymbol{\nabla} X$ is a theorem in every $T-F$-system. $\forall X \boldsymbol{\nabla} X$ is equivalent with $\forall X \neg \nabla X$, which is equivalent with $\forall X \neg(\diamond X \wedge \diamond \neg X)$, which is equivalent with $\forall X(\square X \vee \triangleright X)$ (everything is either necessary or impossible), which is equivalent with $\forall X(\square X \vee \square \neg X)$ (everything is necessarily true or necessarily false). So, to prove that $\forall X \boldsymbol{\nabla} X$ is a theorem in every $T-F$-system, we show that $\forall X(\square X \vee \square \neg X)$ is a theorem in every $T-F$-system.

$$
\begin{gathered}
\forall X(\square X \vee \square \neg X) \text {. Everything is non-contingent. } \\
(1) \neg \forall X(\square X \vee \square \neg X), 0 \\
(2) \exists X \neg(\square X \vee \square \neg X), 0[1, \neg \forall] \\
(3) \neg(\square P \vee \square \neg P), 0[2, \exists] \\
(4) \neg \square P, 0[3, \neg \vee] \\
(5) \neg \square \neg, 0[3, \neg \vee] \\
(6) \diamond \neg P, 0[4, \neg \square] \\
(7) \diamond \neg \neg P, 0[5, \neg \square] \\
(8) 0 r 1[7, \diamond] \\
(9) \neg \neg P, 1[7, \diamond] \\
(10) P, 1[9, \neg \neg] \\
(11) 0 r 2[6, \diamond] \\
(12) \neg P, 2[6, \diamond] \\
\text { (13) } 1=2[8,11, T-F] \\
\text { (14) } P, 2[10,13, T-I i] \\
(15) *[12,14]
\end{gathered}
$$

Note that $\exists X(\diamond X \wedge \diamond \neg X)$ is true in a possible world $\omega$ only if there are at least two possible worlds that are accessible from $\omega$, one in which $X$ is true and one in which $X$ is false (for some $X$ ). But according to $C-F$, every possible world can see at most one possible world.

Here are some other theorems that include the contingency and noncontingency operators and that can be proved in every system: $\forall X(\nabla X \leftrightarrow \nabla \neg X)$ (for every $X: X$ is contingent iff the negation of $X$ is contingent), $\forall X(\boldsymbol{\nabla} X \leftrightarrow$ 
$\boldsymbol{\nabla} \neg X$ ) (for every $X: \quad X$ is non-contingent iff the negation of $X$ is noncontingent), $\forall X(\square X \vee \nabla X \vee \backslash)$ (everything is either necessary, contingent or impossible), nothing is both necessary and contingent $(\neg \exists X(\square X \wedge \nabla X))$, and nothing is both contingent and impossible $(\neg \exists X(\nabla X \wedge \wedge X))$.

We cannot show that nothing is both necessary and impossible in every system in this paper, but $\neg \exists X(\square X \wedge \diamond X)$ is a theorem in every system that includes $T$ or $D$. If there is something that is necessary and impossible, then everything is necessary and impossible; $\exists X(\square X \wedge \diamond X) \rightarrow \forall X(\square X \wedge \diamond X)$ is a theorem in every system in this paper.

If everything is either necessary, contingent or impossible and not everything is necessary or impossible, then obviously something is contingent. And if everything is either necessary, contingent or impossible and something is contingent, then obviously not everything is necessary or impossible.

We have shown that something is necessary, that something is impossible and that something is contingent, given the existence postulate. Since nothing is both necessary and contingent, nothing is both contingent and impossible and nothing is both necessary and impossible (given $T$ or $D$ ), we can see that there are at least three non-equivalent propositions if we assume the existence postulate (and our underlying logic includes $T$ or $D$ ). However, given the existence postulate we can prove something stronger, namely that there are at least four distinct propositions: something that is necessary, something that is true but not necessary, something that is false but not impossible and something that is impossible. Before we prove this, let us first note that $\neg \exists X \exists Y \exists Z(\neg(X \Leftrightarrow Y) \wedge \neg(X \Leftrightarrow Z) \wedge \neg(Y \Leftrightarrow Z))$ is derivable from $\forall X(\square X \vee \neg \diamond X)$ in every system (it is left to the reader to verify this claim). If everything is non-contingent, there are no more than two distinct propositions. Recall that the distinctions between what is true, necessary and possible collapse and that the distinctions between what is false, impossible and possibly false collapse if we assume $C-F$ (and $C-T$ ) and that all necessary propositions are necessarily equivalent and that all impossible propositions are necessarily equivalent. Since we also have observed that there are at least two distinct propositions, we can conclude that there are exactly two distinct propositions according to some systems.

Assume that the existence postulate holds. Then there are at least four distinct propositions (at least in every $T$-system). In other words, we have that $\exists X \exists Y \exists Z \exists W(\neg \square(X \leftrightarrow Y) \wedge \neg \square(X \leftrightarrow Z) \wedge \neg \square(X \leftrightarrow W) \wedge \neg \square(Y \leftrightarrow$ $Z) \wedge \neg \square(Y \leftrightarrow W) \wedge \neg \square(Z \leftrightarrow W))$ is derivable from $\exists X \exists Y(\neg \square(X \rightarrow$ 
$Y) \wedge \neg \square(X \rightarrow \neg Y))$. To prove this claim, we will first establish that there is something that is true but not necessarily true $(\exists X(X \wedge \neg \square X))$, or —in other words - that there is something that is true but possibly false $(\exists X(X \wedge$ $\diamond \neg X))$, and that there is something that is false but not impossible $(\exists X(\neg X \wedge$ $\neg \backslash X)$ ), or -in other words - that there is something that is false but possibly true $(\exists X(\neg X \wedge \diamond X))$. I will prove the first proposition and leave the second to the reader. Since we have shown that the existence postulate is equivalent to the proposition that there is something contingent, we have established the desired result if we can show that $\exists X(X \wedge \neg \square X)$ is derivable from $\exists X(\diamond X \wedge \diamond \neg X)$. Let us now prove this.

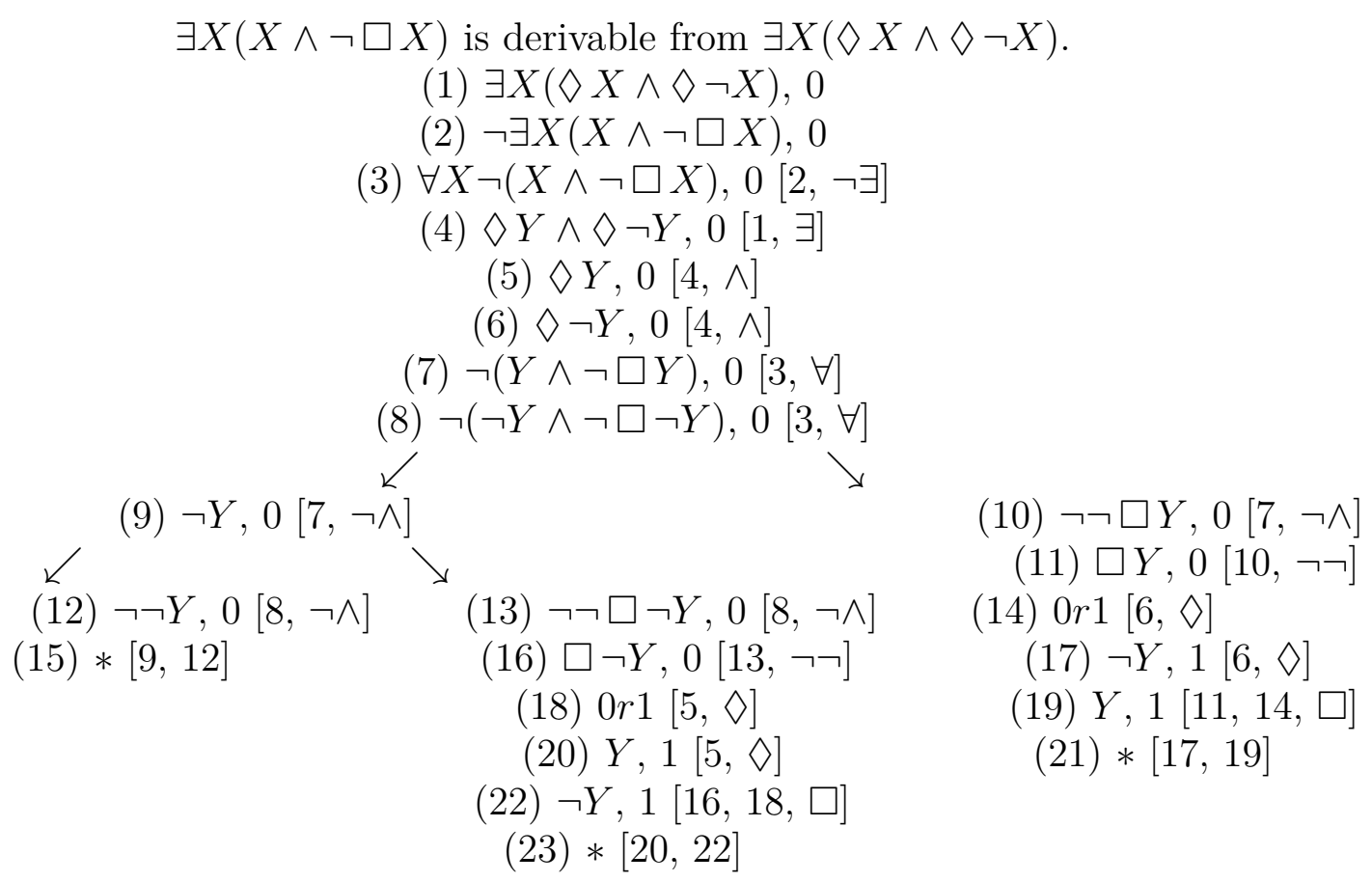

From $\exists X(X \wedge \neg \square X)$ we can derive the proposition that there is something contingently true (true and contingent) in every system that includes $D$ (or $T)$, that is, the following formula is derivable in all $D$ - and $T$-systems: $\exists X(X \wedge$ $\nabla X)$, which is equivalent with $\exists X(X \wedge(\diamond X \wedge \diamond \neg X))$. Likewise, $\exists X(\neg X \wedge$ $\nabla X)$ (which is equivalent with $\exists X(\neg X \wedge(\diamond X \wedge \diamond \neg X))$ is derivable from $\exists X(\neg X \wedge \neg \diamond X)$ in all $D$ - and $T$-systems. According to $\exists X(\neg X \wedge \nabla X)$, there is something contingently false (false and contingent). Hence, there is something that is necessary, there is something that is true but not necessary, there is something that is false but not impossible, and there is something that is impossible. Now, we can use these propositions as premises in an argument 
for our conclusion, that is, we can show that $\exists X \exists Y \exists Z \exists W(\neg \square(X \leftrightarrow Y) \wedge$ $\neg \square(X \leftrightarrow Z) \wedge \neg \square(X \leftrightarrow W) \wedge \neg \square(Y \leftrightarrow Z) \wedge \neg \square(Y \leftrightarrow W) \wedge \neg \square(Z \leftrightarrow W))$ is derivable from $\exists X \square X, \exists X(X \wedge \neg \square X), \exists X(\neg X \wedge \triangleright X)$ and $\exists X \square \neg X$. Since all these premises are derivable from the existence postulate, it follows that the proposition that there are at least four distinct propositions is derivable from the existence postulate. It is left to the reader to verify these claims (assume that the system includes $T$ ).

Let us conclude this section by mentioning some other things that can be derived from the existence postulate (in systems with $T$ ). Given the existence postulate, we can prove that there are at least three distinct propositions which are possible, that is, the following formula is derivable from the existence postulate: $\exists X \exists Y \exists Z((\diamond X \wedge \diamond Y \wedge \diamond Z) \wedge \neg \square(X \leftrightarrow Y) \wedge \neg \square(X \leftrightarrow Z) \wedge$ $\neg \square(Y \leftrightarrow Z))$. To prove this proposition we can use the following premises: $\exists X \square X, \exists X(X \wedge \neg \square X)$ and $\exists X(\neg X \wedge \triangleright X)$. Furthermore, given the existence postulate, we can prove that there are at least three distinct propositions which are possibly false (not necessarily true), that is, the following formula is derivable from the existence postulate: $\exists X \exists Y \exists Z((\diamond \neg X \wedge \diamond \neg Y \wedge \diamond \neg Z) \wedge$ $\neg \square(X \leftrightarrow Y) \wedge \neg \square(X \leftrightarrow Z) \wedge \neg \square(Y \leftrightarrow Z))$. To prove this proposition we can use the following premises: $\exists X(X \wedge \neg \square X), \exists X(\neg X \wedge \diamond X)$ and $\exists X \square \neg X$. This might suggest that there are at least six distinct propositions. However, we cannot show this.

One of the main reasons behind the existence postulate for Lewis was that he wanted a postulate that categorically distinguished the system(s) of strict implication from that of material implication. And given the existence postulate we can show that strict implication does not collapse into material implication. In other words, if we accept the existence postulate we can show that there is some $X$ and some $Y$ such that $X$ materially implies $Y$ even though $X$ does not necessarily imply $Y$, that is $\exists X \exists Y((X \rightarrow Y) \wedge \neg \square(X \rightarrow Y))$ is derivable from $\exists X \exists Y(\neg \square(X \rightarrow Y) \wedge \neg \square(X \rightarrow \neg Y))$. Since we have shown that the existence postulate is equivalent with $\exists X(\diamond X \wedge \diamond \neg X)$, we will use this formula in our derivation.

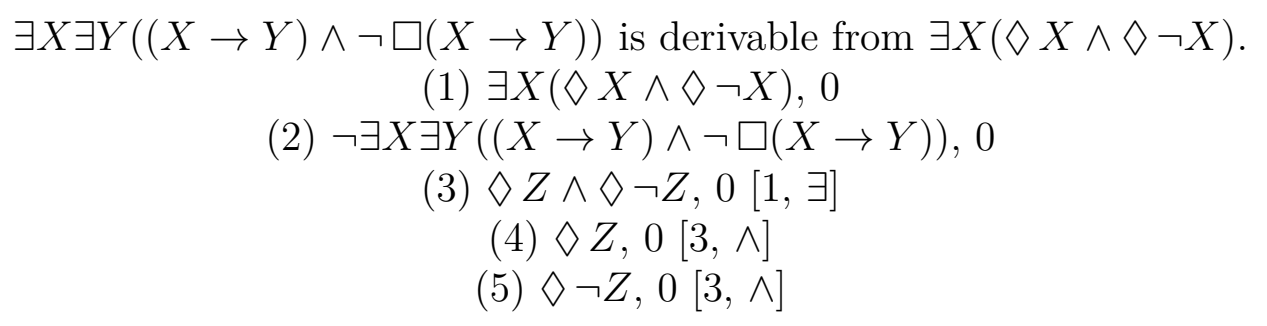




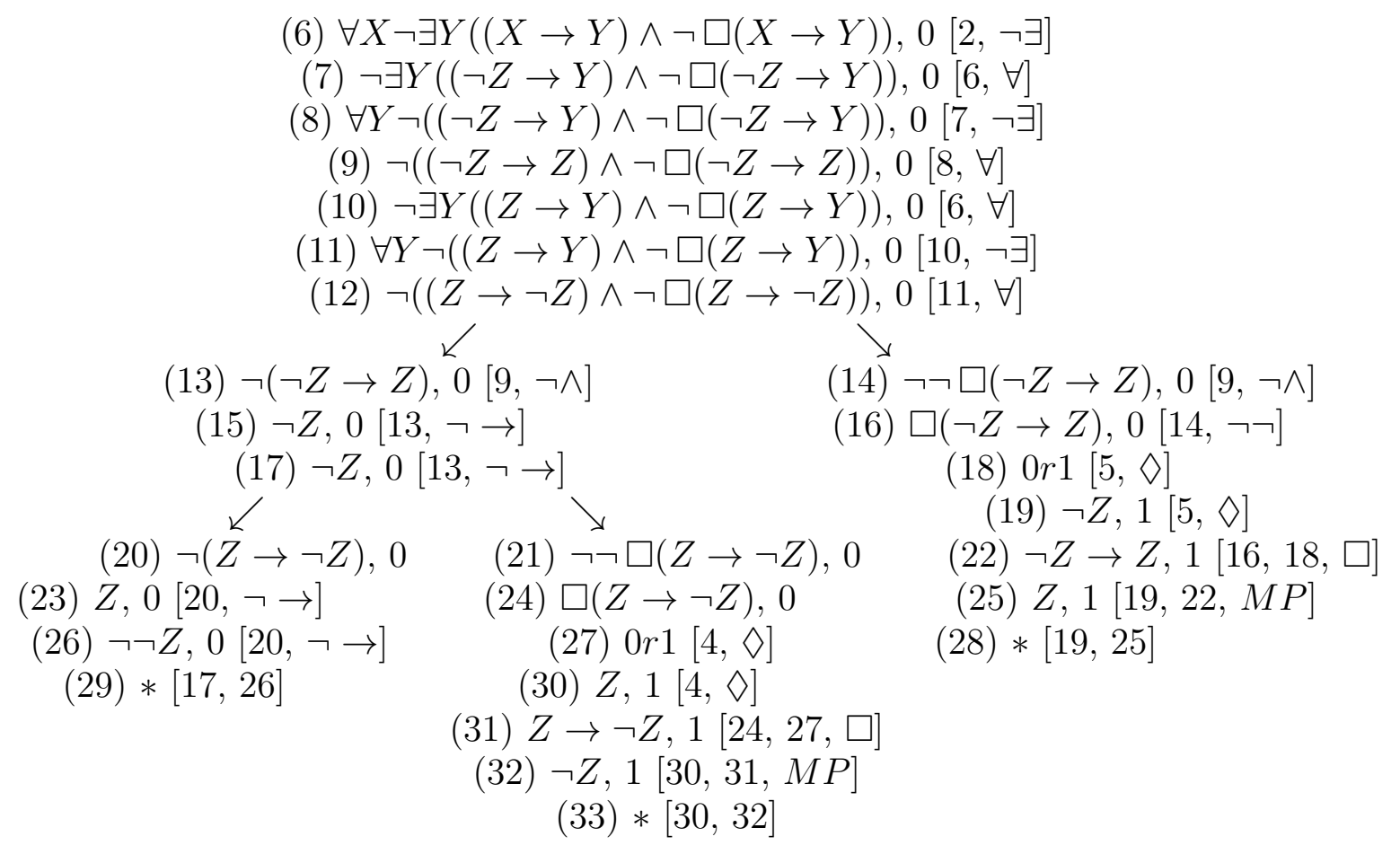

\section{$5 \quad$ Soundness and completeness}

All the normal modal tableau systems (without propositional quantifiers) in this paper are sound and complete with respect to their semantics. In this section, I will go through the main steps in the soundness and completeness proofs. Then, I will show how to modify the proofs to establish that the extended systems are sound. It is an open question whether or not the extended systems are complete. ${ }^{4}$

Let $S=T-X_{1}, \ldots, T-X_{n}$ be a normal modal tableau system as defined above (see Section 4.1). Then we shall say that the class of models, $\mathfrak{M}$, corresponds to $S$ iff $\mathfrak{M}=\mathfrak{M}\left(C-X_{1}, \ldots, C-X_{n}\right)$.

$S$ is sound with respect to $\mathfrak{M}$ iff $\Gamma \vdash_{S} A$ entails $\mathfrak{M}, \Gamma \Vdash A . S$ is complete with respect to $\mathfrak{M}$ iff $\mathfrak{M}, \Gamma \Vdash A$ entails $\Gamma \vdash_{S} A$.

\footnotetext{
4 The soundness and completeness proofs for the normal modal tableau systems in this section are similar to some soundness and completeness proofs that can be found in Priest (2008), chapters 2 and 3.
}

Organon F 26 (3) 2019: 505-536 


\subsection{Soundness theorems}

Let $\mathcal{M}=\langle\mathfrak{W}, \mathfrak{R}, \mathfrak{v}\rangle$ be a model and let $\mathfrak{B}$ be a branch in a tableau. Then $\mathfrak{B}$ is satisfiable in $\mathcal{M}$ iff there is a function $f$ from $0,1,2, \ldots$ to $\mathfrak{W}$ such that (i) $A$ is true in $f(i)$ in $\mathcal{M}$, for every node $A, i$ on $\mathfrak{B}$, (ii) if $i r j$ is on $\mathfrak{B}$, then $\mathfrak{R} f(i) f(j)$ in $\mathcal{M}$, (iii) if $i=j$ is on $\mathfrak{B}$, then $f(i)$ is $f(j)$. If $f$ satisfies these conditions, we say that $f$ shows that $\mathfrak{B}$ is satisfiable in $\mathcal{M}$.

(Soundness lemma I). If the branch $\mathfrak{B}$ is satisfiable in the model $\mathcal{M}$ and a tableau rule is applied to it, then it produces at least one extension, $\mathfrak{B}^{\prime}$, such that $\mathfrak{B}^{\prime}$ is satisfiable in $\mathcal{M}$.

Proof. The proof is by induction on the height of the derivation. Let $f$ be a function that shows that the branch $\mathfrak{B}$ is satisfiable in $\mathcal{M}$.

Connectives. Straightforward.

Modal operators. Suppose that $\square A, i$ and $i r j$ are on $\mathfrak{B}$ and that we apply $(\square)$ to $\mathfrak{B}$. Then we get an extension, $\mathfrak{B}^{\prime}$, of $\mathfrak{B}$ that includes $A, j$. Since $\mathfrak{B}$ is satisfiable in $\mathcal{M}, \square A$ is true in $f(i)$. Furthermore, for any $i$ and $j$ such that $\operatorname{irj}$ is on $\mathfrak{B}, \mathfrak{R} f(i) f(j)$. Hence, $A$ is true in $f(j)$. Suppose that $\diamond A, i$ is on $\mathfrak{B}$ and that we apply $(\diamond)$ to $\mathfrak{B}$. Then we obtain an extension, $\mathfrak{B}^{\prime}$, of $\mathfrak{B}$ that contains nodes of the form $\operatorname{irj}$ and $A, j$. Since $\mathfrak{B}$ is satisfiable in $\mathcal{M}, \diamond A$ is true in $f(i)$. Hence, for some $\omega$ in $\mathfrak{W}, \mathfrak{R} f(i) \omega$ and $A$ is true in $\omega$. Let $f^{\prime}$ be the same as $f$ except that $f^{\prime}(j)=\omega$. Since $f$ and $f^{\prime}$ differ only at $j, f^{\prime}$ shows that $\mathfrak{B}$ is satisfiable in $\mathcal{M}$. Moreover, by definition, $\mathfrak{R} f^{\prime}(i) f^{\prime}(j)$ and $A$ is true in $f^{\prime}(j)$. Hence, $f^{\prime}$ shows that $\mathfrak{B}^{\prime}$ is satisfiable in $\mathcal{M}$.

$(\neg \square)$ and $(\neg \diamond)$. Similar.

$(C U T)$ and the identity rules. $C U T$ is obviously sound since every sentence is either true or false in a possible world. Suppose that $A(i)$ and $i=j$ are on $\mathfrak{B}$ and that we apply $T-I i$ to get $A(j)$. Then $f(i)=f(j)$, for $\mathfrak{B}$ is satisfiable in $\mathcal{M}$. If $A(i)$ is $B, i$, then $B$ is true in $f(i)$. Accordingly, $B$ is true in $f(j)$, as required. The other cases are similar.

Accessibility rules. I will consider one example to illustrate the method. $(T-F)$. Suppose that $i r j$ and $i r k$ are on $\mathfrak{B}$ and that we apply $(T-F)$ to $\mathfrak{B}$ to get an extension that contains $i=j$. Since $\mathfrak{B}$ is satisfiable in $\mathcal{M}, \mathfrak{R} f(i) f(j)$ and $\mathfrak{R} f(i) f(k)$. Hence, $f(j)=f(k)$, for $\mathfrak{R}$ satisfies $(C-F)$. Other steps: similar.

(Soundness theorem I). Every normal modal system in this paper is sound with respect to its semantics. 
Proof. Suppose that $B$ does not follow from $\Gamma$ in $\mathfrak{M}$, where $\mathfrak{M}$ is the class of models that corresponds to $S$. Then every premise in $\Gamma$ is true and the conclusion $B$ false in some world $\omega$ in some model $\mathcal{M}$ in $\mathfrak{M}$. Consider an $S$-tableau whose initial list consists of $A, 0$ for every $A$ in $\Gamma$ and $\neg B, 0$, where ' 0 ' refers to $\omega$. Then the initial list is satisfiable in $\mathfrak{M}$. Every time we apply a rule to this list it produces at least one extension that is satisfiable in $\mathfrak{M}$ (by the Soundness Lemma). Accordingly, we can find a whole branch such that every initial section of this branch is satisfiable in $\mathfrak{M}$. If this branch is closed, then some sentence is both true and false in some possible world in $\mathcal{M}$ in $\mathfrak{M}$. Nevertheless, this is impossible. Hence, the tableau is open. Accordingly, $B$ is not derivable from $\Gamma$ in $S$. Consequently, if $B$ is derivable from $\Gamma$ in $S$, then $B$ follows from $\Gamma$ in $\mathfrak{M}$.

\subsection{Completeness theorems}

In this section, I will show that all the normal modal tableau systems (without propositional quantifiers) in this paper are complete with respect to their semantics. However, first I will introduce some important concepts.

Intuitively, a complete branch is a branch where every rule that can be applied has been applied and a complete tableau is a tableau where every rule that can be applied has been applied. There can be several different (complete) tableaux for the same sentence or set of sentences, since the tableau rules may be applied in different orders. To produce a complete tableau, we can use the following method (which is usually not the simplest one). (1) For every open branch on the tree, one at a time, start from its root and move towards its tip. Apply any rule that produces something new to the branch. For example, $(\diamond)$ is applied at most once to a node of the form $\diamond A, i$. We do not apply any rules to a branch that is closed. Some rules may have several possible applications, for example, $\square$. Then we make all applications at once. (2) When we have done this for all open branches on the tree, we repeat the procedure. $(T-D)$ introduces a new possible world. If a rule introduces a new possible world, it is applied once at the tip of every open branch at the end of every cycle when we have dealt with all nodes. If there is still something to do according to this method, the tableau is incomplete; if not, it is complete.

(Induced Model). Let $\mathfrak{B}$ be an open complete branch of a tableau, let $i, j, k$, etc. be numbers on $\mathfrak{B}$, and let $I$ be the set of numbers on $\mathfrak{B}$. We 
shall say that $i \sim j$ just in case $i=j$, or ' $i=j$ ' or ' $j=i$ ' occurs on $\mathfrak{B}$. $\sim$ is an equivalence relation and $[i]$ is the equivalence class of $i$. The model $\mathcal{M}=\langle\mathfrak{W}, \mathfrak{R}, \mathfrak{v}\rangle$ induced by $\mathfrak{B}$ is defined as follows. $\mathfrak{W}=\left\{\omega_{[i]}: i \in I\right\}, \mathfrak{R} \omega_{[i]} \omega_{[j]}$ iff $\operatorname{irj}$ occurs on $\mathfrak{B}$. Let $P$ be a propositional variable. Then, if $P, i$ occurs on $\mathfrak{B}$, then $P$ is true in $\omega_{[i]}$, that is, $\omega_{[i]}$ is an element in $\mathfrak{v}(P)$, and if $\neg P, i$ occurs on $\mathfrak{B}$, then $P$ is false in $\omega_{[i]}$, that is, $\omega_{[i]}$ is not an element in $\mathfrak{v}(P)$.

If our tableau system does not include $T-F, \sim$ is reduced to identity and $[i]=\{i\}$. Hence, in such systems, we may take $\mathfrak{W}$ to be $\left\{\omega_{i}: i\right.$ occurs on $\left.\mathfrak{B}\right\}$ and dispense with the equivalence classes.

(Completeness Lemma). Let $\mathfrak{B}$ be an open branch in a complete tableau and let $\mathcal{M}$ be a model induced by $\mathfrak{B}$. Then, for every formula $A$ :

(i) if $A, i$ is on $\mathfrak{B}$, then $A$ is true in $\omega_{[i]}$, and

(ii) if $\neg A, i$ is on $\mathfrak{B}$, then $A$ is false in $\omega_{[i]}$.

Proof. The proof is by induction on the complexity of $A$.

(i) Atomic formulas. The result is true by definition.

Connectives. I will consider two cases to illustrate the method. $(\wedge)$ Suppose $A$ is of the form $B \wedge C$. Then $(\wedge)$ has been applied to $B \wedge C, i$ and we have $B, i$ and $C, i$ on $\mathfrak{B}$. By the induction hypothesis, $B$ is true in $\omega_{[i]}$, and $C$ is true in $\omega_{[i]}$. It follows that $B \wedge C$ is true in $\omega_{[i]}$, as required. ( $\vee$ ) If $A$ is of the form $B \vee C$, then $(\vee)$ has been applied to $B \vee C, i$. Hence, either $B, i$ or $C, i$ is on $\mathfrak{B}$. By the induction hypothesis, either $B$ is true in $\omega_{[i]}$ or $C$ is true in $\omega_{[i]}$. It follows that $B \vee C$ is true in $\omega_{[i]}$, as required. Other cases. Similar.

Modal operators. ( $\square$ ). Suppose that $A$ is of the form $\square B$ and that $\square B, i$ is on $\mathfrak{B}$. Then for all $j$ such that $\operatorname{ir} j$ is on $\mathfrak{B}, B, j$ is on $\mathfrak{B}$. By the induction hypothesis, for all $\omega_{[j]}$ such that $\mathfrak{R} \omega_{[i]} \omega_{[j]}, B$ is true in $\omega_{[j]}$. Consequently, $\square B$ is true in $\omega_{[i]}$, as required. $(\diamond)$ Suppose that $A$ is of the form $\diamond B$ and that $\diamond B, i$ is on $\mathfrak{B}$. Then for some $j$, ir $j$ and $B, j$ are on $\mathfrak{B}$. By the induction hypothesis, $\mathfrak{R} \omega_{[i]} \omega_{[j]}$ and $B$ is true in $\omega_{[j]}$. It follows that $\diamond B$ is true in $\omega_{[i]}$, as required.

(ii) Atomic formulas. The result is true by definition.

Connectives. I will consider one example to illustrate the method. $(\neg \vee)$ Suppose $A$ is of the form $\neg(B \vee C)$. Then $(\neg \vee)$ has been applied to $\neg(B \vee C), i$ and we have $\neg B, i$ and $\neg C, i$ on $\mathfrak{B}$. By the induction hypothesis, $B$ is false in $\omega_{[i]}$, and $C$ is false in $\omega_{[i]}$. It follows that $B \vee C$ is false in $\omega_{[i]}$, as required. Other cases. Similar.

Modal operators. $(\neg \square) A$ is of the form $\neg \square B$. Suppose that $\neg \square B, i$ is 
on $\mathfrak{B}$. Then $\diamond \neg B, i$ is on $\mathfrak{B}$. Hence, for some $j, \operatorname{ir} j$ and $\neg B, j$ are on $\mathfrak{B}$. By the induction hypothesis, $\mathfrak{R} \omega_{[i]} \omega_{[j]}$ and $B$ is false in $\omega_{[j]}$. It follows that $\square B$ is false in $\omega_{[i]}$, as required. $(\neg \diamond)$. Similar.

(Completeness Theorem I). Every normal modal system in this paper is complete with respect to its semantics.

Proof. First we prove that the theorem holds for our weakest system $K$. Then we extend the theorem to all normal modal systems that are stronger than $K$. Let $\mathfrak{M}$ be the class of models that corresponds to $K$, that is, $\mathfrak{M}$ is the class of all models.

Suppose that $B$ is not derivable from $\Gamma$ in $K$. Then it is not the case that there is a closed $K$-tableau whose initial list comprises $A, 0$ for every $A$ in $\Gamma$ and $\neg B, 0$. Let $t$ be a complete $K$-tableau whose initial list comprises $A, 0$ for every $A$ in $\Gamma$ and $\neg B, 0$. Then $t$ is not closed, $t$ is open. Hence, there is at least one open branch in $t$. Let $\mathfrak{B}$ be an open branch in $t$. The model induced by $\mathfrak{B}$ makes all the premises in $\Gamma$ true and $B$ false in $\omega_{[0]}$. Accordingly, it is not the case that $B$ follows from $\Gamma$ in $\mathfrak{M}$. Consequently, if $B$ follows from $\Gamma$ in $\mathfrak{M}$, then $B$ is derivable from $\Gamma$ in $K$.

To prove that all normal modal extensions of $K$ are complete with respect to their semantics, we have to check that the model induced by the open branch $\mathfrak{B}$ is of the right kind. To do this we first check that this is true for every single semantic condition. Then we combine each of the individual arguments. I will go through one step to illustrate the method.

$C-F$. Suppose that $\mathfrak{R} \omega_{[i]} \omega_{[j]}$ and $\mathfrak{R} \omega_{[i]} \omega_{[k]}$. Then $i r j$ and irk occur on $\mathfrak{B}$ [by the definition of an induced model]. Since $\mathfrak{B}$ is complete, $(T-F)$ has been applied and $j=k$ occurs on $\mathfrak{B}$. Hence, $j \sim k$. So, $[j]=[k]$. It follows that $\omega_{[j]}=\omega_{[k]}$, as required [by the definition of an induced model].

\subsection{Soundness for systems with propositional quantifiers}

In the extended systems, a tableau branch can contain propositional quantifiers. Hence, we must modify the soundness proof slightly.

(Soundness lemma II). If the branch $\mathfrak{B}$ is satisfiable in the model $\mathcal{M}$ and Organon F 26 (3) 2019: 505-536 
a tableau rule is applied to it, then there is a model $\mathcal{M}^{\prime}$ and an extension of $\mathfrak{B}, \mathfrak{B}^{\prime}$, such that $\mathfrak{B}^{\prime}$ is satisfiable in $\mathcal{M}^{\prime}$.

Proof. Most cases are trivial modifications of the steps in the proof for soundness lemma I in Section 5.1 (just let $\mathcal{M}^{\prime}$ be $\mathcal{M}$ ). The only really interesting new steps are the steps for the quantifiers.

$(\forall)$. Straightforward.

$(\exists)$. Suppose that $\exists X A, i$ is on $\mathfrak{B}$ and that we apply $(\exists)$ to $\mathfrak{B}$. Then we get an extension, $\mathfrak{B}^{\prime}$, of $\mathfrak{B}$ that includes $A[Y / X], i$, where $Y$ is a propositional variable new to the branch. Since $\mathfrak{B}$ is satisfiable in $\mathcal{M}, \exists X A$ is true in $f(i)$. Hence, there is some sentence $B$ in $\mathcal{L}$ such that $B$ is substitutable for $X$ in $A$ and $A[B / X]$ is true in $f(i)$. Let $\mathcal{M}^{\prime}=\left\langle\mathfrak{W}, \mathfrak{R}, \mathfrak{v}^{\prime}\right\rangle$ be like $\mathcal{M}$, except that $\mathfrak{v}^{\prime}(Y)=\left\{f(j): \mathcal{M}^{\prime}, f(j) \Vdash B\right\}$. Then $A[Y / X]$ is true in $f(i)$ in $\mathcal{M}^{\prime}$.

(Soundness theorem II). All augmented systems are sound with respect to their semantics.

Proof. Once soundness lemma II is established, the rest of the proof is an easy modification of the soundness proof for soundness theorem I.

\subsection{An example of a countermodel}

In this section, I will consider one example of a countermodel that proves that a certain sentence is not valid and hence that it is not a theorem in a certain system. This will illustrate how one can use countermodels to prove invalidity and 'non-theoremhood'.

The following sentence is a theorem in every (extended) system that includes $T-D$ (or $T-T): \forall X(\square X \rightarrow \diamond X) . \forall X(\square X \rightarrow \diamond X)$ says that everything that is necessary is possible. But this formula is not a theorem in every system. It is, for example, not a theorem in the smallest extended system $K_{E x t}$. To show this, we will first establish that the sentence is not valid in the class of all models. The following model establishes this: $\mathcal{M}=\langle\mathfrak{W}, \mathfrak{R}, \mathfrak{v}\rangle$, where $\mathfrak{W}=\omega, \mathfrak{R}$ is empty, and $P$ is true in $\omega$ (or false; it does not matter). Since no possible world is accessible from $\omega$, it is vacuously true that $P$ is true in every possible world that is accessible from $\omega$. Hence, $\square P$ is true in $\omega$. $\diamond P$ is true in $\omega$ only if $P$ is true in some possible world that is accessible from $\omega$. Since no possible world is accessible from $\omega$, it is not the case that $P$ is true in some possible world that is accessible from $\omega$. Accordingly, $\diamond P$ is not true in $\omega$. It follows that $\square P \rightarrow \diamond P$ is false in $\omega$, for the antecedent 
is true and the consequent is false in $\omega . \forall X(\square X \rightarrow \diamond X)$ is true in $\omega$ only if $(\square X \rightarrow \diamond X)[P / X]$ is true in $\omega$. But $(\square X \rightarrow \diamond X)[P / X]=(\square P \rightarrow \diamond P)$ and $\square P \rightarrow \diamond P$ is false in $\omega$. Therefore, $\forall X(\square X \rightarrow \diamond X)$ is not true in $\omega$. Consequently, $\forall X(\square X \rightarrow \diamond X)$ is not valid in the class of all models. We have shown that the system $K$ (and $K_{E x t}$ ) is sound with respect to the class of all models (Sections 5.1 and 5.3). It follows that $\forall X(\square X \rightarrow \diamond X)$ is not a theorem in $K_{E x t}$.

\section{Acknowledgements}

I would like to thank the editors and two anonymous reviewers for some valuable comments on an earlier version of this paper and Matteo Pascucci for some interesting discussions on the topic of propositional quantification.

\section{References}

Barcan (Marcus), Ruth. 1946. "A Functional Calculus of First Order Based on Strict Implication." Journal of Symbolic Logic 11(1): 1-16. https://doi .org/10.2307/2269159

Blackburn, Patrick, de Rijke, Maarten and Venema, Yde. 2001. Modal Logic. Cambridge: Cambridge University Press. https://doi.org/10.1017/CB09781107050884

Blackburn, Patrick, van Benthem, Johann and Wolter, Frank (Editors). 2007. Handbook of Modal Logic. Amsterdam: Elsevier.

Bull, Robert. 1969. "On Modal Logic with Propositional Quantifiers." Journal of Symbolic Logic 34(2): 257-263. https://doi .org/10.2307/2271102

Carnap, Rudolf. 1946. "Modalities and Quantification." Journal of Symbolic Logic 11, 2 , 33-64. https://doi.org/10.2307/2268610

Chellas, Brian. 1980. Modal Logic: An Introduction. Cambridge: Cambridge University Press.

Corsi, Giovanna. 2002. "A Unified Completeness Theorem for Quantified Modal Logics." Journal of Symbolic Logic, Vol. 67, No. 4, 1483-1510. https://doi.org/10.2178/jsl/1190150295

D’Agostino, Marcello, Gabbay, Dov, Hähnle, Reiner and Posegga, J. (Editors). 1999. Handbook of Tableau Methods. Dordrecht: Kluwer Academic Publishers.

Fine, Kit. 1970. "Propositional Quantifiers in Modal Logic." Theoria 36(3): 336-346. https://doi.org/10.1111/j.1755-2567.1970.tb00432.x

Fitting, Melvin and Mendelsohn, Richard. 1998. First-Order Modal Logic. Dordrecht: Kluwer.

Organon F 26 (3) 2019: 505-536 
Gabbay, Dov. M. 1971. "Montague Type Semantics for Modal Logics with Propositional Quantifiers." Mathematical Logic Quarterly 17(1): 245-249. https://doi.org/10.1002/malq.19710170128

Gallin, Daniel. 1975. Intensional and Higher-Order Modal Logic: With Applications to Montague Semantics. Amsterdam: North-Holland.

Garson, James. 1984. "Quantification in Modal Logic." In Handbook of Philosophical Logic Vol. 2 (2nd edition 3, 2001), edited by D. M. Gabbay, and F. Guenthner, 267-323. Dordrecht: Springer.

Garson, James. 2006. Modal Logic for Philosophers. New York: Cambridge University Press.

Hintikka, Jaakko. 1961. "Modality and Quantification." Theoria 27(3): 117-128. https://doi.org/10.1111/j.1755-2567.1961.tb00020.x

Hughes, George E. and Cresswell, Max J. 1968. An Introduction to Modal Logic. London: Routledge. Reprinted 1990.

Hughes, George E. and Cresswell, Max J. 1996. A New Introduction to Modal Logic. London: Routledge. Reprinted 2004.

Jeffrey, Richard. 1967. Formal Logic: Its Scope and Limits. New York: McGraw-Hill.

Kaplan, David. 1970. "S5 with quantifiable propositional variables." Journal of Symbolic Logic 35(2): 355.

Kracht, Marcus. 1999. Tools and Techniques in Modal Logic. Number 142 in Studies in Logic. Amsterdam: Elsevier.

Kripke, Saul. 1959. "A Completeness Theorem in Modal Logic." Journal of Symbolic Logic 24(1), 1-14. https://doi.org/10.2307/2964568

Lewis, Clarence Irving and Langford, Cooper Harold. 1932. Symbolic Logic. New York: Dover Publications. Second edition 1959.

Parks, Zane. 1976. "Investigations into Quantified Modal Logic-I." Studia Logica 35(2): 109-125. https://doi.org/10.1007/BF02120875

Priest, Graham. 2008. An Introduction to Non-Classical Logic. Cambridge: Cambridge University Press. https://doi .org/10.1017/CB09780511801174

Smullyan, Raymond. 1968. First-Order Logic. Heidelberg: Springer-Verlag.

Stalnaker, Robert and Thomason, Richmond. 1968. "Abstraction in First-Order Modal Logic." Theoria 34(3): 203-207. https://doi.org/10.1111/j.1755-2567.1968.tb00351.x

Thomason, Richmond and Stalnaker, Robert. 1968. "Modality and Reference." Nô̂s 2(4): 359-372. https://doi.org/10.2307/2214461

Thomason, Richmond. 1970. "Some Completeness Results for Modal Predicate Calculi." In Philosophical Problems in Logic, edited by K. Lambert, 56-76, Dordrecht: Reidel. 